DATE: JUL 12 199?

REPLY TO
ATTN OF: Office of Environmental Guidance:Whitehead:6-6073

SUBJECT: DOE Environment, Environmental Restoration \& Waste Management Field Organization Directory

TO: Distribution

The purpose of this memorandum is to provide Program and Operations Offices with a copy of a document entitled: "DOE Environment, Environmental Restoration and Waste Management Field Organization Directory." This directory was developed by the Office of Environmental Guidance, RCRA/CERCLA Division (EH-231) from an outgrowth of the Departments efforts to identify and establish the regulatory response lead persons in the Field Organizations. ${ }^{1}$ The directory was developed for internal EH-231 use to identify both the DOE and DOE contractor Field Organizations in the Environment, Environmental Restoration and Waste Management areas.

The Field Organization directory is divided into three substantive sections: (1) Environment; (2) Environmental Restoration; and (3) Waste Management which are organized to correspond to the management hierarchy at each Field Organization. The information provided includes the facility name and address, individual managers name, and telephone/fax numbers.

A draft of the directory was sent to DOE elements for review and correction via a December 7,1992 , memorandum. We greatly appreciated the assistance provided by the field in compiling the directory. As a result of the interest expressed in the directory we are providing copies to Program Offices and Field Organizations. If such interest continues, EH-231 will update the directory biannually.

Corrections or modifications to the directory may be provided to Beverly Whitehead of my staff at (202) 586-6073. For additional copies contact the Office of Scientific and Technical Information (OSTI) at (615) 576-8401.

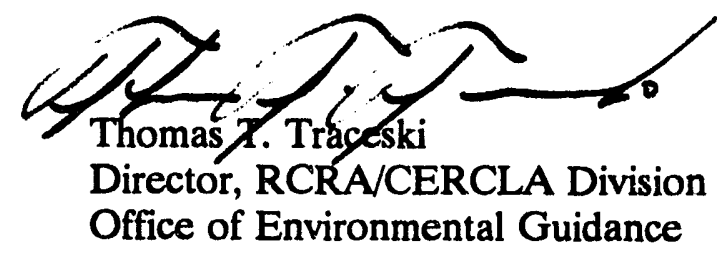

Attachment

${ }^{1}$ EH-231 memorandum, dated February 6, 1992, subject: "Identification of RCRA/CERCLA/ TSCA Regulatory Response Leads." 


\section{Preren Olixes}

O.J. Lawrence, CE-64 A Knight, CP-20

Henry Garson, DP-3.2

Lake Barreth, DP-6.1

Dan Rhoades, DP-6.2

Charles Halsted, DP-27

Donald Xnuth, DP-60

Thomas Evana, DP.62

Fritz Wolf, DP-624

John Ford. DP-63

Karl Goodwin, DP-636

Richard Hahn, DP-64

Rendy Lynch, DP-643

Ralph Hagan, DP-644

Ken Ferlic, DP-65

M. Whitaker, DR-1

Lynne Fairobent, EH-1

Joe Fitzgerald, EH-30

Harry Pettengill, EH-40

Rick Joner, EH-41

Richard Moore, EI-23.1

Jim Disbrow, EI-523

Paul Grimm, EM-2

Tom Elsasser, EM-4

Ellen Livingston-Behan, EM-5

Willis Bisty, EM-6

Randal Scoth EM-20

Jill Lytic, EM-30

Jim Turi, EM-33

Lee Stevens, EM-331

Mark Frei, EM-34

Joseph Coleman, EM-35

Jay Rhoderick, EM-351

Kent Hancock, EM-352

John Tseng EM-36

Jack Baubliz, EM-40

Jim Fiore. EM-42

Bill Wisenbaker, EM-43

Sally Mann, EM-44

Mark Stahr, EM-441

Ralph Lightner, EM-45

J. A. Ahlquist, EM-452

Clyde Frank, EM-50

Tom Evans, EM-53

Larry Weiner, EM-60

Kitty Gandee, EM-62

Don Donaldson, EM-121

David Moves, EP.63

James Farley, ER-8.2

Frederick Koomanoff, ER-10

Albert Evans, ER-13

Sat Goel, ER-14

David Goodwin, ER-20.1

Omer Goktepe, ER.22

Joseph MoGrory, ER-23

John Yatea, ER-42

Steven Rousi, ER-53

William Osburn, ER-73

Robert Wood. ER-74

James Carney, ER-912

Craig Zamuda, FE-6

N. L Johnson, FE-33

Mark Matarrese, FE-64

David Jeweth, FE-222

Hal Delaplane, FE-423

William Deanison, GC-11

Charles Bradley, NE-33

Charles Thompson, NE-43

$R_{\text {aj Sharma, NE-47 }}$

Robert Gisch, NE-60

Sim Pairobeat, OE-11

Pranklin Petern, RW-2

Steve Brocoum, RW-22

Dwight Shelor, RW-30

D. M. Valentine, RW-332

Gerald Parker, RW-333

A.G. Joceph, ST-30
Rodney Adelman, WDC

\section{Field Oaticen}

N. S. Dienes, Albuquerque Operations Office P.M. Ramey, Albuquerque Operations Office Rich Sena, Albuquerque Operations Office

C. L. Soden, Albuquerque Operations Office

R. E. Sabre, Albuquerque Operations Office

Mike Butler, Brookhaven Area Office

D.J. Cook, Central Training Academy, Al

M. Flannigan, Chicago Operations Office

Joel Haugen, Chicago Operations Office

J. Hunze, Chicago Operations Office

Paul Neeson, Chicago Operations Office

John Kennedy, Chicago Operations Office

T. J. Rowland, Fernald Operations Office

G. Westerbeck, Fernald Operations Office

Jerry Lyle, Idaho Operations Office

Gerald C Bowman, Idaho Operations Officee

Walt Sato, Idaho Operations Office

Peggy Hinman, Idaho Operations Office

Bruce Church, Nevada Operations Office

Don Elle, Nevada Operations Office

Joe Fiore, Nevada Operations Office

Jim Magruder, Nevada Operations Office

Steve Mellington, Nevada Operations Office

J. D. Ross, Nevada Operations Office

Rodney R. Nelson, Oak Ridge Operations Office

H. Wayne Hibbilts, Oak Ridge Operations Office

Robert Sleeman, Oak Ridge Operations Office

Peter Gross, Oak Ridge Operations Office

L.K. Price, Oak Ridge Operations Office

Larry Radcliff, Oak Ridge Operations Office

Don Alexanter, Richland Operations Office

J. Bauer, Ric land Operations Office

Rudy Guercia, Richland Operations Office

R.D. Izatt, Richland Operations Office

Bob Holt, Richland Operations Office

Leo Little, Richland Operations Office

J. Hartman, Rocky Flats Office

Frazer Lockhart, Rocky Flats Office

Tom Lukow, Rocky Flats Office

Al Pauole, Rocky Flats Office

Mark Van Derpuy, Rocky Flats Office

E. Ballard, San Francisco Operations Office

J. Cullen, San Francisco Operations Office

Jim Davis, San Francisco Operations Office

Alex Dong, San Francisco Operations Office

Joe Juetten, San Francisco Operations Office

Terry Vaeth, San Francisco Operations Office

Thomas Heenan, Savannah River Operations Office

Lou Goidell, Savannah River Operations Office

A.B.Gould, Savannah River Operations Office

Lenard Sjostrom, Savannah Operations Office

M.G. O'Rear, Savannah River Operations Office

Steve Wright, Savannah River Operations Office

R. B. Provencher, West Valley Project Office

Rob Waldman, Alaska Power Administration

Alex Crawley, Bartlesville Project Office

Thomas Wesson, Bartlesville Project Office

Alexandra Smith, Bonneville Power Administration, AJ

Thru: BPA, RM 8G033

John Ganz, Morgantown Energy Technology Center

Joseph Martin, Morgantown Energy Technology

Center

Jim Killen, Naval Petroleum Reserves in California

D.J. Newquist, Naval Petroleum Reserves in Wyoming, Utah, \& Colorado, Casper, WY

Melvin Keller, Pittsburgh Energy Technology Center

Earl Shollenberger, Pittsburgh Naval Reactors Office,

Thru: NE-60
Andrew Seepo, Schenectady Naval Reactors Office, Thru: NE-60

Jim Lloyd, Southeastern Power Administration

Don Hayes, Southwestern Power Administration

Melissa Smith, Strategic Petroleum Reserve Project Management Office

Vicki Ponce, Western Power Administration

Gerald Johnson, Manager, Amarillo Area Office

(AAO)

James Morley, Manager, Dayton Area Office (DAO)

Earl Bean, Manager, Kansas City Area Office (KCAO)

Kathleen Carison, Manager, Kirtland Area Office (KAO)

Jerry Bellows, Acting Manager, Los Alamos Area Office (LAAO)

Richard E. Glass, Manager, Pinellas Area Office (PAO)

J. E. Solecki, Manager, Grand Junction Project Office

Arlen Hunt, Waste Isolation Pilot Plant Project Office (WIPP-AL)

James Mewhinney, WIPP Project Office (WIPP-AL)

A. R. Chernoff, Jr., Project Manager, Uranium Mill Tailings

Remedial Action Project Office

Kathleen Grassmeir, Yucca Mountain Project Office

W.R. Miller, Special Projects Manager, ES\&H Division, Morgantown, WV

Paul K. Kearns, Manager, NREL, Golden Operations Office

Jeff Baker, NREL Area Office

Nat Brown, SSCPO

\section{cc: Other Organizations}

National Low-Level Waste Management Program, EG\&G Idaho Hazardous Waste Remedial Action Program, (HAZWRAP)

Remedial Action Program Information Center 


\section{Rerulatory Contects}

Rob Waldman, Alaska Power Administration Constance Soden, Albuquerque Field Office Chris Gentile, Allied-Signal, Inc. Scott White, Allied-Signal, Inc. Gerald Johnson, Amarillo Area Office Donna Green, Argonne Area Office (East)

Greg Bass, Argonne Area Office (West)

David Alleman, Bartlesville Project Office

Jon Cooper, Batavia Area Office

Stephen Sander, Bonneville Power Administration

Mike Butler, Brookhaven Area Office

Paul Neeson, Chicago Field Office

James Johnson, Dayton Area Office

Charles Friedman, EG \& G Mound Applied Technologies

Roger Liddle, Energy Technology Engineering Center

Herbert Feely, Environmental Measurements Laboratory

Don Cossairt, FERMI National Accelerator Laboratory

Walter Quaider, Fernald Field Office

Bennett Young, Grand Junction Project Office

Lisa Green, Idaho Field Office

Margaret Hinman, Idaho Field Office

David Caughey, Kansas City Area Office

George Lasker, Kirtland Area Office

Richard Frounfelker, K-25 Site Office

Steve Slaten, Los Alamos Area Office

John Ganz, Morgantown Energy Technology Center

Jeffrey Baker, NREL Area Office

Gary Walker, Naval Petroleum Reserve (California)

David Miles, Naval Petroleum Reserve (Wyoming, Colorado, \& Utah)

Donald Elle, Nevada Field Office

Stephen Mellington, Nevada Test Site Office

Margaret Lachman, New Brunswick Laboratory

Margaret Wilson, Oak Ridge Field Office

Connor Matthews, Oak Ridge National Laboratory Site Office

David Tidwell, Paducah Site Office

David Ingle, Pinellas Area Office

Elias George, Pittsburgh Energy Technology Center

Earl Shollenberger, Pittsburgh Naval Reactors Office

Melda Rafferty, Portsmouth Site Office

Allen Wrigley, Princeton Area Office

Jim Scott, Princeton University Plasma Physics Laboratory

Julie Erickson, Richland Field Office

Rudy Guercia, Richland Field Office

Robert Holt, Richland Field Office

Frazer Lockhart, Rocky Flats Office

Joe Wienand, Rocky Flats Office

Edward Ballard, San Francisco Field Office

Joseph Cullen, San Francisco Field Office

Ronald Peterson, Savannah River Field Office

Andrew Seepo, Schenectady Naval Reactors Office

Jim Lloyd, Southeastern Power Administration

David Dossett, Southwestem Power Administration

Melissa Smith, Strategic Petroleum Reserve

Tony Robledo, Superconducting Super Collider Project Office

Charlene Esparza-Baca, UMTRA Project Office

Alan Gibson, Weldon Spring Site Office

Steve McCracken, Weldon Spring Site Office

Ken Mathias, Western Area Power Administration

Nancy Harris, Westinghouse Electric Corporation

Richard Provencher, West Valley Demonstration Project Office

James Mewhinney, WIPP

Wendy Dixon, Yucca Mountain Project Office

Brian DeMonia, Y-12 Site Office

Autar Rampertaap, EM-45/TREV

Tom Evans, EM-55/GTN 


\section{Environment, Environmental Restoration,}

and

Waste Management

Dield Organization Directory

\section{Juily 1993}

Propersed by

U.S. Dephartinent CI Innergy

Qffice of Emvirommental Guidance

RCRA/GrRota Division

UTIERIII)

Wadington, DC

Technical Stipport by

Argonne Nationai Laboratory MIMTR
Washington, DC 


\section{TABLE OF CONTENTS}

PAGE

NUMBER

\section{ALBUQUERQUE FIFLD OFFICE (AL)}

- Albuquerque Field Office - DOE

Sandia National Laboratories - Albuquerque (SNLA)

$A L-1$

Sandia National Laboratories - Livermore (SNLL)

$A L-3$

$A L-4$

- Amarillo Area Office - DOE

Mason \& Hanger - Silas Mason Co., Inc.

$A L-5$

$A L-6$

- Dayton Area Office - DOE

EG \& G Mound Applied Technologies

$A L-8$

$A L-9$

- Grand Junction Project Office - DOE Chem-Nuclear Geotech

$A L-10$

$A L-11$

- Kansas City Area Office - DOE

$A L-13$ Allied Signal Inc.

$A L-14$

- Kirtland Area Office - DOE

$A L-15$

- Los Alamos Area Office - DOE

$A L-16$

Los Alamos National Laboratory (LANL)

$A L-17$

- Pinellas Area Office - DOE

AL. -18

Martin Marietta Specialty Components, Inc. - Pinellas Plant

$A L-19$

- Uranium Mill Tailings (UMTRA) Project Office - DOE

$A L-20$ Jacobs Engineering Group MK-Ferguson Co.

$A L-21$

$A L-22$

- Waste Isolation Pilot Plant (WIPP) - DOE

$A L-23$ Westinghouse WIPP Project

$A L-24$

\section{CHICAGO FIELD OFFICE (CH)}

- Chicago Field Office - DOE Ames Laboratory (lowa State University)

$\mathrm{CH}-1$

$\mathrm{CH}-2$

- Argonne Area Office (East) - DOE

Argonne National Laboratory (ANL-East)

$\mathrm{CH}-3$

$\mathrm{CH}-5$

- Argonne Area Office (West) - DOE

Argonne National Laboratory (ANL-West)

$\mathrm{CH}-6$

$\mathrm{CH}-7$

- Batavia Area Office - DOE

$\mathrm{CH}-8$

Fermi National Accelerator Laboratory (FERMILAB)

$\mathrm{CH}-9$ 
- Brookhaven Area Office - DOE

$\mathrm{CH}-10$

Brookhaven National Laboratory (BNL)

$\mathrm{CH}-11$

- Environmental Measurements Laboratory - DOE

$\mathrm{CH}-12$

- National Renewable Energy Laboratory Area Office - DOE

$\mathrm{CH}-13$

National Renewable Energy Laboratory (NREL - formerly SERI)

$\mathrm{CH}-14$

- New Brunswick Laboratory - DOE

$\mathrm{CH}-15$

- Princeton Area Office - DOE

$\mathrm{CH}-16$

Princeton University Plasma Physics Laboratory (PPPL)

$\mathrm{CH}-17$

- Solar Energy Research Institute (SERI - See National Renewable Energy Laboratory - NREL)

\section{ENERGY TECHNOLOGY CENTERS (ETC)}

- Bartlesville Project Office - DOE

ETC - 1

- Morgantown Energy Technology Center (METC) - DOE

ETC - 2

EG\&G WASC Inc. - Morgantown

ETC - 3

- Pittsburgh Energy Technology Center (PETC) - DOE

ETC - 4

\section{FERNALD FIELD OFFICE (FN)}

- Fernald Field Office - DOE

Westinghouse Environmental Management Co. of Ohio

FN - 1

FN -2

IDAHO FIELD OFFICE (ID)

- Idaho Field Office - DOE

Babcock \& Wilcox/INEL

ID - 1

EG\&G Idaho, Inc.

ID - 3

Westinghouse Idaho Nuclear Co., Inc.

ID - 5

ID -6

- West Valley Demonstration Project Office - DOE

ID - 8

\section{NAVAL PETROLEUM RESERVE (NOSR)}

- Naval Petroleum Reserve (California) - DOE

NOSR - 1

Bechtel Petroleum Operations, Inc.

NOSR - 2

- Naval Petroleum and Oil Shale Reserve (Wyoming, Colorado \& Utah) - DOE NOSR - 3 


\section{NEVADA FIELD OFFICE (NV)}

- Nevada Field Office - DOE

EG\&G Energy Measurements Inc.

NV - 1

NV -2

- Nevada Test Site Office - DOE

NV -4

Reynolds Electrical \& Engineering Co. Inc.

NV - 5

- Pacific Area Support Office - DOE

NV -7

\section{OAK RIDGE FIELD OFFICE (OR)}

- Oak Ridge Field Office - DOE

Martin Marietta Energy Systems, Inc. (MMES) - Central Staff Offices

OR - 1

OR -3

- Oak Ridge National Laboratory Site Office - DOE

OR -6

Oak Ridge National Laboratory (ORNL)

OR -7

- K-25 Site Office - DOE

K-25 Site - MMES

OR - 9

OR - 1

- Y-12 Site Office - DOE

OR -12

Y-12 Plant - MMES

OR -13

- Paducah Site Office - DOE

OR -15

Paducah Gaseous Diffusion Plant (PGDP) - MMES

OR -16

- Portsmouth Site Office - DOE

OR -17

Portsmouth Gaseous Diffusion Plant (PORTS) - MMES

OR -18

- Formerly Utilized Sites Remedial Action Program (FUSRAP) - DOE

OR -19

Bechtel National Inc.

OR - 20

- Weldon Spring Site Office - DOE

Weldon Spring Site - MK-Ferguson Company

OR -21

OR - 22

\section{PITTSBURGH NAVAL REACTORS (PNR)}

- Pittsburgh Naval Reactors Office - DOE

Westinghouse Electric Corp. - Bettis Atomic Power Laboratory

PNR - 1

PNR - 2

\section{POWER ADMINISTRATIONS (PA)}

- Alaska Power Administration

PA - 1

- Bonneville Power Administration

PA - 2

- Southeastern Power Administration

PA - 4

- Southwestern Power Administration

PA - 5

- Western Area Power Administration

PA - 6 
RICHLAND FIELD OFFICE (RL)

- Richland Field Office - DOE RL - 1

Batelle Memorial Institute Pacific Northwest Laboratories (PNL) RL - 3

Westinghouse Hanford Co. $\quad$ RL - 5

ROCKY FLATS (RF)

- Rocky Flats Office - DOE RF - 1

EG\&G Rocky Flats $\quad$ RF - 3

SAN FRANCISCO FIELD OFFICE (SF)

- San Francisco Field Office - DOE SF - 1

Lawrence Berkeley Laboratory (LBL) SF - 3

Lawrence Livermore National Laboratory (LLNL) SF - 4

Stanford Linear Accelerator Center (SLAC) SF - 5

- Energy Technology Engineering Center (ETEC) - DOE SF - 7 Energy Technology Engineering Center Rockwell International - $\quad$ SF - 8

Rocketdyne Division

SAVANNAH RIVER FIELD OFFICE (SR)

- Savannah River Field Office - DOE SR - 1

Westinghouse Savannah River Co. SR - 3

SCHENECTADY NAVAL REACTORS (SNR)

- Schenectady Naval Reactors Office - DOE SNR - 1

STRATEGIC PETROLEUM RESERVE (SPR)

- Strategic Petroleum Reserve (DOE) SPR - 1

DymMcDermott Petroleum Operations $\quad$ SPR - 2

SUPERCONDUCTING SUPER COLLIDER (SSC)

- Superconducting Super Collider Project Office - DOE SSC - 1

Superconducting Super Collider Laboratory $\quad$ SSC - 2

YUCCA MOUNTAIN PROJECT OFFICE (YMPO)

- Yucca Mountain Site Characterization Project Office - DOE 
Albuquerque Field Office (AL) 
ALBUQUERQUE FIELD OFFICE - DOE

U.S. DOE

PENNSYLVANIA \& H STREET

KIRTLAND AFB, NEW MEXICO 87116

P.O. BOX 5400

ALBUQUERQUE, NEW MEXICO 87185-5400

FAX* COMMEF.CIAL

MANAGER: BRUCE TWINING

$845-4420$

505/845-6049

\section{ENVIRONMENT}

ENVIRONMENT, SAFETY \& HEALTH

Assistant Manager: Dennis Krenz

$845-4665$

$505 / 845-6121$

ENVIRONMENTAL PROTECTION DIVISION

Director: Constance Soden ${ }^{\star \star}$

845-6714 505/845-5586

\section{ENVIRONMENTAL RESTORATION}

OFFICE OF ENERGY \& SPECIAL PROGRAMS

Assistant Manager: James Bickel

$845-4665 \quad 505 / 845-4829$

ENVIRONMENTAL RESTORATION PROJECT OFFICE

Project Manager: Richard Sena

845-4239

$505 / 845-4887$

\section{WASTE MANAGEMENT}

OPERATIONS AND WEAPONS

Assistant Manager: Stephen Guidice

$845-4837$

$505 / 845-6038$

WASTE MANAGEMENT \& OPERATIONAL SURETY DIVISION

Director: Nicholas Dienes

845-4664 505/845-5230

WASTE MANAGEMENT BRANCH

Chief: Oren Critchfield

845-4664 505/845-5656

*DIAL AREA CODE

* RCRA/CERCLA/TSCA REGULATORY RESPONSE LEAD

$A L-1$ 
SANDIA NATIONAL LABORATORIES - ALBUQUERQUE (SNLA)

1515 EUBANK BOULEVARD, SE

ALBUQUERQUE, NEW MEXICO 87123

P.O. BOX 5800

ALBUQUERQUE, NEW MEXICO 87185

FAX*

PRESIDENT: AL NARATH

INTERNAL PROGRAMS DIVISION:

VICE PRESIDENT: GLEN CHENEY
$844-1120$

$844-8617$

844-3415

Director: Joseph Stiegler

ES\&H PROGRAM MANAGEMENT CENTER

Director: James Baremore

ENVIRONMENT

ES\&H DIRECTORATE

844-0636

$505 / 845-9887$

\section{ENVIRONMENTAL RESTORATION}

\section{ES\&H DIRECTORATE}

Director: Joseph Stiegler

844-0636

$505 / 844-7221$

ES\&H PROGRAM ER/WM PMO

Manager: Thomas Blejwas

848-0999

$505 / 848-0905$

ENVIRONMENTAL RESTORATION PROGRAM

Program Manager: Warren Cox

848-0999

505/848-0911

ENVIRONMENTAL RESTORATION DEPARTMENT I

Department I Manager: Francis Nimick

848-0417

$505 / 848-0427$

ENVIRONMENTAL RESTORATION DEPARTMENT ॥

Department II Manager: Joseph Fernandez

848-0417

$505 / 848-0462$ 


\section{WASTE MANAGEMENT}

ES\&H DIRECTORATE

Director: Joseph Stiegler

844-3415 505/845-8889

ES\&H PROGRAM ER/WM PMO

Manager: Thomas Blejwas

848-0999 505/848-0905

WASTE MANAGEMENT PROGRAM

Manager: Stephen Ward

848-0999 505/848-0488

HAZARDOUS WASTE DEPARTMENT

Manager: James Yeager

848-0493 505/848-0491

POLLUTION PREVENTION DEPARTMENT

Manager: Jimmy Fish

848-0998 505/848-0984

RADIOACTIVE \& MIXED WASTE DEPARTMENT

Manager: John Guth

848-0999 505/848-0912

**RCRA/CERCLA/TSCA REGULATORY RESPONSE LEAD

$A L \cdot 3$ 
SANDIA NATIONAL LABORATORIES - LIVERMORE (SNL/CA)

7011 EAST AVENUE

LIVERMORE, CALIFORNIA 94550

P.0. 30X 969

LIVERMORE, CALIFORNIA 94550

FAX *

COMMERCIAL

VICE PRESIDENT: JOHN CRAWFORD

294-1516

$510 / 294-2211$

\section{ENVIRONMENT}

ENVIRONMENTAL PROTECTION DEPARTMENT

Manager: Don Nissen

294-3418

$510 / 294-2767$

ENVIR NMENTAL MONITORING PROGRAM

Dave Brekke

294-3418

$510 / 294-2233$

ENVIRONMENTAL RESTORATION

ENVIRONMENTAL PROTECTION DEPARTMENT

Manager: Don Nissen

294-3418

$510 / 294-2767$

ENVIRONMENTAL ASSESSMENT AND RESTORATION

Sandy Leo

294-3418

$510 / 294-1476$

\section{WASTE MANAGEMENT}

ENVIRONMENTAL PROTECTION DEPARTMENT

Manager: Don Nissen

$294-3418$

$510 / 294-2767$

WASTE MANAGEMENT PROGRAM

Kim Shepodel

294-3418

$510 / 294-1475$

WASTE MINIMIZATION

Alice Johnson-Duarte

$294-\uparrow 418$

$510 / 294-3266$

*DIAL AREA CODE

**RCRA/CERCLA/TSCA REGULATORY RESPONSE LEAD 


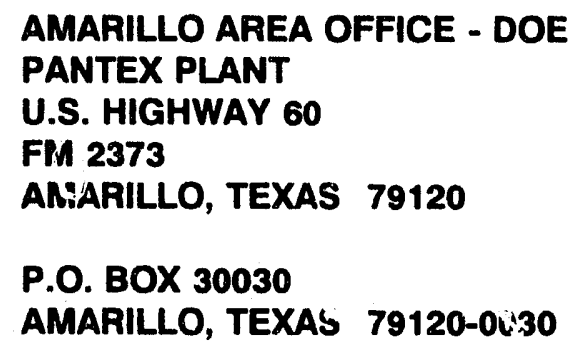

FAX*

477-3185 COMMERCIAL

AREA MANAGER: GERALD JOHNSON**

\section{ENVIRONMENT}

Assistant Area Manager For Compliance: David Heim

ES\&H MANAGEMENT BRANCH

Acting Chief: Anthony Ladino

\section{ENVIRONMENTAL RESTORATION}

Assistant Manager for Compliance: David Heim

OPERATIONAL SURETY MANAGEMENT BRANCH

Lead Engineer: Lawrence Paradee

Environmental Projects Coordinator: Daniel Ferguson

477-3185

$806 / 477-3125$

477-3185

806/477-3169

806/477-3180

477-5864

477-5864

806/477-3161

806/477-3126

\section{WASTE MANAGEMENT}

Assistant Manager for Compliance: David Heim

477-3185

$806 / 477-3125$

OPERATIONAL SURETY MANAGEMENT BRANCH

Chief: Lawrence Paradee

477-5864

806/477-3161

Engineer: John Thurston
477-5864 
MASON \& HANGER - SILAS MASON CO., INC.

PANTEX PLANT

17 MILES EAST OF AMARILLO, HIGHWAY 60

AMARILLO, TEXAS 79177

P.O. BOX 30020

AMARILLO, TEXAS 79177

FAX* COMMERCIAL

PLANT MANAGER:

Richard Loyhry (Mason \& Hanger)

477-4105

$806 / 477-6200$

\section{ENVIRONMENT}

ENVIRONMENT, SAFETY, HEALTH \& WASTE MANAGEMENT (BATELLE PANTEX)

Director: John Burr

477-5518 $\quad 806 / 477-5200$

ENVIRONMENTAL PROTECTION DEPARTMENT

Manager: Rudy Taylor

477-5641

$806 / 477-5104$

ENVIRONMENTAL COMPLIANCE

Section Manager: William Bowman

$477-5641$

$806 / 477-4403$

ENVIRONMENTAL MONITORING

Section Manager: Dan McGrath

477-5641

$806 / 477-5567$

\section{ENVIRONMENTAL RESTORATION}

ENVIRONMENT, SAFETY, HEALTH \& WASTE MANAGEMENT

Director: John Burr

$477-5518$

$806 / 477-5200$

WASTE MANAGEMENT DEPARTMENT

Manager: Gary Baker

477-5641

$806 / 477-4440$

ENVIRONMENTAL RESTORATION

Section Manager: Michelle Lincoln

$477-5641$

806/477-5942

* Dial area code

*\#CRA/CERCLA/TSCA REGULATORY RESPONSE LEAD

$A L \cdot 6$ 
ENVIRONMENT, SAFETY, HEALTH \& WASTE MANAGEMENT

Director: John Burr

477-5518

$806 / 477-5200$

WASTE MANAGEMENT DEPARTMENT

Manager: Gary Baker

477-5641

$806 / 477-4440$

WASTE OPERATIONS MANAGEMENT

Section Manager: Billy Faubion

477-5447

806/477-3514

*DIAL AREA CODE

**RCRA/CERCLA/TSCA REGULATORY RESPONSE LEAD

$A L=7$ 
DAYTON AREA OFFICE - DOE

1 MOUND ROAD

MIAMISBURG, OHIO 45343

P.O. BOX 66

MIAMISBURG, OHIO 45343-0066

FAX

COMMERCIAL

MANAGER: LARRY KIRKMAN (Acting)

865-4489

$513 / 865-3271$

DEPUTY AREA MANAGER: WARREN SHERAD (Acting)

865-4489 513/865-3252

\section{ENVIRONMENT}

ENVIRONMENT, SAFETY, HEALTH \& COMPLIANCE BRANCH

Chief: Michael Reker

865-4489

$513 / 865-3252$

General Engineer: James Johnson**

865-4489 513/865-5234

\section{ENVIRONMENTAL RESTORATION}

ENVIRONMENT, SAFETY, HEALTH \& COMPLIANCE BRANCH

Chief: Michael Reker

865-4489

$513 / 865-3252$

ENVIRONMENTAL RESTORATION

Arthur Kleinrath

865-4489

$513 / 865-3597$

WASTE MANAGEMENT

PROGRAMS \& OPERATIONAL SURETY BRANCH

Chief: Dewaine Eckman

$865-4489$

$513 / 865-3487$

WASTE MANAGEMENT

Timothy Frazier

865-4489

$513 / 865-3748$

**RCRA/CERCLA/TSCA REGULATORY RESPONSE LEAD

$A L-8$ 
EG \& G MOUND APPLIED TECHNOLOGIES

1 MOUND ROAD

MIAMISBURG, OHIO $\mathbf{4 5 3 5 3 - 3 0 0 0}$

P.O. BOX 3000

MIAMISBURG, OHIO 45343-3000

FAX* COMMERCIAL

GENERAL MANAGER: DONALD MICHEL

865-3742 513/865-5090

\section{ENVIRONMENT}

ES\&H, ER \& WM DEPARTMENT

Vice President: Warren Smith

865-3099 513/865-3752

ENVIRONMENTAL TECHNOLOGY \& MONITORING

Supervisor: Chris Kinard

865-3725 513/865-3775

\section{ENVIRONMENTAL RESTORATION}

ES\&H, ER \& WM DEPARTMENT

Vice President: Warren Smith

865-3099

$513 / 865-3752$

ENVIRONMENTAL RESTORATION/CERCLA

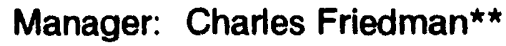

865-3835

$513 / 865-4541$

\section{WASTE MANAGEMENT}

ES\&H, ER \& WM DEPARTMENT

Director: Warren Smith

865-3099 513/865-3752

WASTE MANAGEMENT

Manager: Ray Finney

865-3725 513/865-3097

*DIAL AREA CODE

**RCRA/CERCLA/TSCA REGULATORY RESPONSE LEAD

$A L-9$ 
GRAND JUNCTION PROJECT OFFICE - DOE

2597 B \& 3/4 ROAD

GRAND JUNCTION, COLORADO 81503

P.O. BOX 2567

GRAND JUNCTION, COLORADO 81502-2567

FAX* COMMERCIAL

OFFICE OF ENERGY \& SPECIAL PROGRAMS (DOE-AL)

ASSISTANT MANAGER: JAMES BICKEL

$845-4665 \quad 505 / 845-4829$

GRAND JUNCTION PROJECTS OFFICE

MANAGER: JIM SOLECKI

$248-6040 \quad 303 / 248-6001$

\section{ENVIRONMENT}

ENVIRONMENTAL, SAFETY, HEALTH \& QUALITY ASSURANCE

Director: Bennett Young ${ }^{\star \star}$

248-6040 303/248-6010

ENVIRONMENTAL RESTORATION

ENVIRONMENTAL, SAFETY, HEALTH \& QUALITY ASSURANCE

Director: Bennett Young

$248-6040 \quad 303 / 248-6010$

WASTE MANAGEMENT

ENVIRONMENTAL, SAFETY, HEALTH \& QUALITY ASSURANCE

Director: Bennett Young

$248-6040 \quad 303 / 248-6010$

*DIAL AREA CODE

**RCRA/CERCLA/TSCA REGULATORY RESPONSE LEAD

$$
A L-10
$$


CHEM-NUCLEAR GEOTECH

2597 B \& 3/4 ROAD

GRAND JUNCTION, COLORADO 81503

P.O. BOX 14000

GRAND JUNCTION, COLORADO 81502

FAX* COMMERCIAL

PRESIDENT: JOHN BOLLIGER

$248-6040 \quad 303 / 248-6400$

\section{ENVIRONMENT}

TECHNICAL

Vice President: Duane Renberger

$248-6040 \quad 303 / 248-6407$

ENVIRONMENTAL COMPLIANCE \& REGULATORY AFFAIRS

Manager: John Winterhalder

$248-6040 \quad 303 / 248-6758$

\section{ENVIRONMENTAL RESTORATION}

TECHNICAL

Vice President: Duane Renberger

$248-6040 \quad 303 / 248-6407$

ENVIRONMENTAL COMPLIANCE \& REGULATORY AFFAIRS

Manager: John Winterhalder

$248-6040 \quad 303 / 248-6758$

OPERATIONS

Vice President: Vincent Tonc

$248-6040 \quad 303 / 248-6464$

ENVIRONMENTAL SUPPORT OPERATIONS

Director: Terry Moore

$248-6040 \quad 303 / 248-6060$

WASTE PROGRAM

Manager: Sam Marutzky

$248-6040 \quad 303 / 248-6059$

*DIAL AREA CODE

**RCRA/CERCLA/TSCA REGULATORY RESPONSE LEAD

$A L-11$ 
WASTE MANAGEMENT

OPERATIONS

Vice President: Vincent Tonc

$248-6040 \quad 303 / 248-6464$

ENVIRONMENTAL SUPPORT OPERATIONS

Director: Terry Moore

$248-6040 \quad 303 / 248-6060$

WASTE MANAGEMENT

Manager: Sam Marutzky

$\cdot 248-6040 \quad 303 / 248-6059$

*DIal area code

*\#RCRA/CERCLA/TSCA REGULATORY RESPONSE LEAD

$A L=12$ 
KANSAS CITY AREA OFFICE - DOE

2000 EAST 95TH STREET

KANSAS CITY, MISSOURI 64131-3095

P.O. BOX 410202

KANSAS CITY, MISSOURI 64141-0202

\begin{tabular}{lcc} 
& FAX & COMMERCIAL \\
\hline AREA MANAGER: EARL BEAN & $997-5059$ & $816 / 997-3341$ \\
ES\&H BRANCH CHIEF: Patrick Hoopes & $997-7310$ & $816 / 997-7003$
\end{tabular}

\section{ENVIRONMENT}

ES\&H BRANCH

Chief: David Caughey

997-7310 816/997-3449

Program Manager: David Caughey**

997-7310 816/997-3449

\section{ENVIRONMENTAL RESTORATION}

ES\&H BRANCH

Chief: David Caughey

997-7310

816/997-3449

Program Manager:

G. Phil Keary

$997-7310$

$816 / 997-7288$

\section{WASTE MANAGEMENT}

ES\&H BRANCH

Chief: David Caughey

$997-7310$

816/997-3449

Program Manager: Margaret Stockdale

997-7310

816/997-7289

\section{*DIAL AREA CODE \\ **RCRA/CERCLA/TSCA REGULATORY RESPONSE LEAD}


ALLIED-SIGNAL, INC.

KANSAS CITY DIVISION

2000 EAST 95TH STREET

KANSAS CITY, MISSOURI 64131

P.O. BOX 419159

KANSAS CITY, MISSOURI 64141-6159

FAX*

PRESIDENT: LOUIS SMITH

ENVIRONMENT

ES\&H DIVISION

Director: Charles Kircher

ENVIRONMENT \& HEALTH PROTECTION

Manager: Dan Stoltz

ENVIRONMENTAL COMPLIANCE

Supervisor: Brad Heacock

RCRA Team Leader: Scott White ${ }^{\star \star}$

CERCLA/TSCA Team Leader: Chris Gentile**

ENVIRONMENTAL APPLICATIONS

ER Team Leader: Dave Brown

\section{ENVIRONMENTAL RESTORATION}

ENVIRONMENTAL RESTORATION/ WASTE MANAGEMENT

Program Manager: Maceo Gray

ER Supervisor: Emerson Brown

WASTE MANAGEMENT

ENVIRONMENTAL RESTORATION/ WASTE MANAGEMENT

Program Manager: Maceo Gray

WM Operations Manager: DeWayne Long

WM Operations Supervisor: Dave Huyett
$997-7257$

$816 / 997-2175$

$997-7257$

$816 / 997-4175$

997-5903

997-5903

997-5903

997-5903

816/997-4034

997-4208

997-4208

816/997-4619

816/997-4906

997-4208 $\quad 816 / 997-4619$

997-7313 816/997-7329

$997-7313 \quad 816 / 997-7304$ 
KIRTLAND AREA OFFICE - DOE

BUILDING 802

1515 EUBANK BOULEVARD, SE

ALBUQUERQUE, NEW MEXICO 87123

P.O. BOX 5400

ALBUQUERQUE, NEW MEXICO 87185-5400

FAX* COMMERCIAL

AREA MANAGER: KATHLEEN CARLSON

$845-6867$

505/845-4094

\section{ENVIRONMENT}

ENVIRONMENTAL, SAFETY, HEALTH AND COMPLIANISE BRANCH

Chief: George Lasker:

$845-4710$

$505 / 845-6869$

\section{ENVIRONMENTAL RESTORATION}

ENVIRONMENTAL, SAFETY, HEALTH AND COMPLIANCE BRANCH

Chief: George Lasker

$845-4710 \quad 505 / 845-6869$

\section{WASTE MANAGEMENT}

ENVIRONMENTAL, SAFETY, HEALTH AND COMPLIANCE BRANCH

Chief: George Lasker

845-4710 505/845-6869

*DIAL AREA CODE

**RCRA/CERCLA/TSCA REGULATORY RESPONSE LEAD

$A L=15$ 
LOS ALAMOS AREA OFFICE - DOE

528 35TH ST.

LOS ALAMOS, NEW MEXICO 87544

FAX*

667-8038

AREA MANAGER: JERRY BELLOWS

\section{ENVIRONMENT}

ENVIRONMENTAL, SAFETY, \& HEALTH BRANCH

Chief: Joe Vozella

ENVIRONMENTAL RESTORATION PROGRAM

Manager: Ted Taylor

ENVIRONMENTAL RESTORATION

ENVIRONMENTAL, SAFETY, \& HEALTH BRANCH

Chief: Joe Vozella

ENVIRONMENTAL RESTORATION PROGRAM

Manager: Steve Slaten**

\section{WASTE MANAGEMENT}

ENVIRONMENTAL, SAFETY, \& HEALTH BRANCH

Chief: Joe Vozella

ENVIRONMENTAL RESTORATION PROGRAM

Manager: Jon Mack
$665-4504$

$505 / 665-5027$

$665-4504$

$505 / 665-7203$

$665-4504$

$505 / 665-5027$

$665-4504$

$505 / 665-5050$

$665-4504$

$505 / 665-5027$

$665-4504$

$505 / 665-5026$ 
LOS ALAMOS NATIONAL LABORATORY (LANL)

LOS ALAMOS, NEW MEXICO 87545

\section{LOS ALAMOS, NEW MEXICO 87545}

DIRECTOR: SIEGFRIED HECKER (MAIL STOP A100)

FAX*

$667-2997$

COMMERCIAL

\section{ENVIRONMENT}

ENVIRONMENTAL MANAGEMENT DIVISION

Leader: Tom Gunderson (MAIL STOP K491)

$665-3811$

$505 / 665-3778$

ENVIRONMENTAL PROTECTION

Group Leader: Kenneth Hargis (MAIL STOP K490)

$667-0486$

$505 / 667-5021$

\section{ENVIRONMENTAL RESTORATION}

ENVIRONMENTAL MANAGEMENT DIVISION

Leader: Tom Gunderson (MAIL STOP K491)

$665-3811$

$505 / 665-3778$

ENVIRONMENTAL RESTORATION

Group Leader: Robert Vocke (MAIL STOP M992)

$667-4747$

$505 / 667-0808$

\section{WASTE MANAGEMENT}

ENVIRONMENTAL MANAGEMENT DIVISION

Leader: Tom Gunderson (MAIL STOP K491)

$665-3811$

$505 / 665-3778$

WASTE MANAGEMENT

Group Leader: Anthony Drypolcher (MAIL STOP E517)

$665-3750$

$505 / 667-7301$ 
PINELLAS AREA OfFICE - DOE

11400 SOUTH BELCHER ROAD

LARGO, FLORIDA 34643

P.O. BOX 2900

LARGO, FLORIDA 34649

FAX *

545-6287

AREA MANAGER: RICHARD GLASS

ENVIRONMENT

ES\&H COMPLIANCE BRANCH

Chief: Dawn Gillas

$541-8370$

$813 / 545-6182$

ENVIRONMENTAL RESTORATION

ENVIRONMENTAL RESTORATION PROGRAM

Manager: David Ingle**

WASTE MANAGEMENT

VIASTE MANAGEMENT PROGRAM

Manager: Gary Schmidtke
$541-8370 \quad 813 / 541-8943$

$541-8370 \quad 813 / 545-6179$

- DIAL AREA code

* RCRA/CERCLA/TSCA REGULATORY RESPONSE LEAD

$A L=18$ 
MARTIN MARIETTA SPECIALTY COMPONENTS, INC.

PINELLAS PLANT

11400 SOUTH BELCHER ROAD

LARGO, FLORIDA 34643

P.O. BOX 2908

LARGC FLORIDA 34649-2908

FAX* COMMERCIAL

GENERAL MANAGER: CHARLES HALL

$545-6347 \quad 813 / 541-8174$

ENVIRONMENT

ENVIRONMENTAL SAFETY \& HEALTH:

Director: Thomas Pharo

$541-8909 \quad 813 / 541-8585$

ENVIRONMENTAL RESTORATION

ENVIRONMENTAL MANAGEMENT

Director: Edward Mayer

$541-8909 \quad 813 / 541-8616$

ENVIRONMENTAL RESTORATION \& PERMITTING

Manager: Charles Biedermann

$541-8909 \quad 813 / 545-6144$

WASTE MANAGEMENT

ENVIRONMENTAL MANAGEMENT

Director: Edward Mayer

$541-8909 \quad 813 / 541-8616$

WASTE MANAGEMENT \& MINIMIZATION

Manager: Delphine Delaineuvilie

541-8909 $\quad 813 / 545-6085$

*DIAL AREA CODE

**RCTA/CERCLA/TSCA REGULATORY RESPONSE LEAD

$A L-19$ 
URANIUM MILL TAILINGS REMEDIAL ACTION (UMTRA) PROJECT OFFICE - DOE FIRST NATIONAL BANK BUILDING

5301 CENTRAL AVENUE, NE

SUITE 1720

ALBUQUERQUE, NEW MEXICO 87108

FAX*

$845-4023$

PROJECT MANAGER: ALBERT CHERNOFF

\section{ENVIRONMENT}

TECHNICAL SUPPORT GROUP

Group Leader: Frank Bosiljevac

ENVIRONMENT AND SAFETY

Manager: Charlene Esparza-Baca *

\section{ENVIRONMENTAL RESTORATION}

TECHNICAL SUPPORT GROUP

Group Leader: Frank Bosiljevac

ENVIRONMENT AND SAFETY

Manager: Charlene Esparza-Baca

\section{WASTE MANAGEMENT}

TECHNICAL SUPPORT GROUP

Group Leader: Frank Bosiljevac

ENVIRONMENT \& SAFETY

Manager: Charlene Esparza-Baca
$845-4023$

$505 / 845-5638$

845-4023

$505 / 845-5664$

$845-4023$

$505 / 845-5638$

$845-4023$

$505 / 845-5664$

$845-4023$

$505 / 845-5638$

$845-4023$

$505 / 845-5664$ 


\section{JACOBS ENGINEERING GROUP}

UMTRA ALBUQUERQUE PROJECT OFFICE

5301 CENTRAL AVENUE, N.E.

SUITE 1700

ALBUQUERQUE, NEW MEXICO 87108

FAX * COMMERCIAL

PROJECT MANAGER: NED LARSON

845-4053

$505 / 845-4030$

\section{ENVIRONMENT}

TECHNICAL SERVICES

Assistant Project Manager: James Gibb

845-4053

$505 / 845-5704$

ENVIRONMENTAL SERVICES

Linda Ulland

$845-4053$

$505 / 845-5671$

\section{ENVIRONMENTAL RESTORATION}

NO ACTIVITY

\section{WASTE MANAGEMENT}

SITE MANAGEMENT

Assistant Project Manager: James Gibb

845-4053

$505 / 845-4011$ 
MK - FERGUSON COMPANY

UMTRA ALBUQUERQUE PROJECT OFFICE

2309 RENALD PLACE, S.E.

SUITE 300

ALBUQUERQUE, NEW MEXICO 87106

P.O. BOX 9136

ALBUQUERQUE, NEW MEXICO 87119

FAX*

$766-3318$

$766-3318$

$766-3318$

Environmental Specialist: John Isham

Manager: John Huff
COMMERCIAL

505/845-5868

\section{ENVIRONMENT}

SAFETY

\section{ENVIRONMENTAL RESTORATION}

SAFETY

Manager: John Huff

Environmental Specialist: John Isham

\section{WASTE MANAGEMENT}

Deputy Proj. Director: Rob Cooney

Operations Manager: Charles Spencer
766-3318 505/845-5868

766-3318 505/845-5868

$766-3318 \quad 505 / 845-5868$
766-3318 505/845-5868 
WASTE ISOLATION PILOT PLANT (WIPP) - DOE

WIPP PROJECT OFFICE

35 MILES EAST OF CARLSBAD

CARLSBAD, NEW MEXICO 88220

P.O. BOX 3090

CARLSBAD, NEW MEXICO 88221

FAX* COMMERCIAL

PROJECT MANAGER: ARLEN HUNT

887-0707 505/887-8108

\section{ENVIAONMENT}

COMPLIANCE BRANCH

Chief: Jerry Reese

$887-0707 \quad 505 / 887-8112$

ENVIRONMENTAL, SAFETY \& HEALTH SECTION

James Mewhinney**

887-0707 505/887-8143

\section{ENVIRONMENTAL RESTORATION}

COMPLIANCE BRANCH

Chief: Jerry Reese

887-0707 505/887-8112

ENVIRONMENTAL, SAFETY \& HEALTH SECTION

James Mewhinney

$887-0707 \quad 505 / 887-8143$

\section{WASTE MANAGEMENT}

TESTING \& OPERATIONS BRANCH

Chief: Micheal McFadden

$887-0707 \quad 505 / 887-8108$

WASTE MANAGEMENT SECTION

Kent Hunter

$887-0707 \quad 505 / 887-8127$ 
WESTINGHOUSE WIPP PROJECT

WIPP ROAD/JAL HIGHWAY

CARLSBAD, NEW MEXICO 88220

P.O. BOX 2078

CARLSBAD, NEW MEXICO 88221

FAX * COMMERCIAL

GENERAL MANAGER: A. LAMAR TREGO

887-5783

505/887-8201

\section{ENVIRONMENT}

ENVIRONMENT, SAFETY \& HEALTH

Manager: Leslie Reed

885-4562 $\quad 505 / 887-8489$

ENVIRONMENTAL \& INDUSTRIAL SAFETY

Manager: Warren Bodily

885-4562 $\quad 505 / 887-8990$

ENVIRONMENTAL COMPLIANCE

Manager: Bryan Howard

885-4562

$505 / 887-8291$

ENVIRONMENTAL MONITORING

Manager: Larry Madl

885-4562

$505 / 887-8400$

\section{ENVIRONMENTAL RESTORATION}

ENVIRONMENT, SAFETY \& HEALTH

Manager: Leslie Reed

885-4562

$505 / 887-8489$

ENVIRONMENTAL \& INDUSTRIAL SAFETY

Manager: Warren Bodily

885-4562

$505 / 887-8990$

*DIAL AREA CODE

**RCRA/CERCLA/TSCA REGULATORY RESPONSE LEAD

$$
A L-24
$$


WASTE MANAGEMENT

OPERATIONS

Assistant General Manager: John Lee

885-6955 505/887-8206 OPERATIONS

Deputy Manager: Harry Bibby

$885-6955 \quad 505 / 887-8266$

WASTE HANDLING OPERATIONS

Clint Kelley

885-6955 505/887-8737

TRANSPORTATION AND HAZARDOUS MATERIAL HANDLING

Jeff Winkel

885-6955 505/887-8234

*DIAL AREA CODE

**RCRA/CERCLA/TSCA REGULATORY RESPONSE LEAD

$A L \cdot 25$ 
Chicago Field Office (CH) 


\section{CHICAGO FIELD OFFICE - DOE 9800 SOUTH CASS AVENUE ARGONNE, ILLINOIS 60439}

FAX*

DEPUTY MANAGER: DAVID GOLDMAN

\section{ENVIRONMENT}

OFFICE OF ASSISTANT MANAGER FOR SAFETY \& SECURITY

Assistant Manager: John Kennedy

ENVIRONMENT, SAFETY \& HEALTH DIVISION

Director: Michael Flannigan

ENVIRONMENTAL PROTECTION BRANCH

Chief: Paul Neeson** (Acting)

\section{ENVIRONMENTAL RESTORATION}

OFFICE OF ASSISTANT MANAGER FOR ENVIRONMENTAL MANAGEMENT AND ENERGY PROJECTS

Assistant Manager: Jefferson Neff (Acting)

252-2654

$708 / 252-2236$

ENVIRONMENTAL RESTORATION DIVISION

Director: Jay Hunze

$252-2654$

$708 / 252-2428$

\section{WASTE MANAGEMENT}

OFFICE OF ASSISTANT MANAGER FOR ENVIRONMENTAL MANAGEMENT AND ENERGY PROJECTS

Assistant Manager: Donald Bray

252-2654

$708 / 252-2235$

WASTE MANAGEMENT AND TECHNOLOGY DEVELOPMENT DIVISION

Director: Joel Haugen
252-2206

252-2206

$708 / 252-2110$

$708 / 252-2110$

$708 / 252-2002$

$708 / 252-2219$

$708 / 252-2258$

252-2835 
AMES LABORATORY (IOWA STATE UNIVERSITY)

109 OFFICE \& LAB BUILDING

AMES, IOWA 50011-3020

FAX*

COMMERCIAL

DIRECTOR: THOMAS BARTON

294-4456

$515 / 294-2770$

\section{ENVIRONMENT}

OPERATIONS DIVISION

Associate Director: Roland Struss

294-4456

$515 / 294-2634$

SAFETY, HEALTH \& PLANT PROTECTION

Manager: Lowell Mathison

294-2155

$515 / 294-7921$

\section{ENVIRONMENTAL RESTORATION}

SCIENCE AND TECHNOLOGY DIVISION

Associate Director: R. Bruce Thompson

294-4456

$515 / 294-9649$

ENVIRONMENTAL TECHNOLOGY DEVELOPMENT PROGRAM

Manager: James Corones (Acting)

294-9683

$515 / 294-9636$

\section{WASTE MANAGEMENT}

SCIENCE \& TECHNOLOGY DIVISION

Associate Director: R. Bruce Thompson

$294-4456$

$515 / 294-9649$

ENVIRONMENTAL TECHNOLOGY DEVELOPMENT PROGRAM

Manager: James Corones (Acting)

294- 5083

$515 / 294-9636$

*DIAL AREA CODE

**RCRA/CERCLA/TSCA REGULATORY RESPONSE LEAD

$\mathrm{CH}-2$ 
ARGONNE AREA OFFICE (EAST) - DOE

9800 SOUTH CASS AVENUE

ARGONNE, ILLINOIS 60439

FAX*

252-2361

MANAGER: ANIBAL TABOAS

\section{ENVIRONMENT}

PROGRAM MANAGEMENI DIVISION:

Director: A. Creig Zook

ENVIRONMENTAL PROGRAMS BRANCH

Chief: Donna Green**

ENVIRONMENTAL COMPLIANCE

Engineer: Yvette Collazo

ENVIRONMENTAL RESTORATION

PROGRAM MANAGEMENT DIVISION:

Director: A. Creig Zook

ENVIRONMENTAL PROGRAMS BRANCH

Chief: Donna Green

ENVIRONMENTAL RESTORATION PROGRAM

Manager: Linda Freeman
252-2361

$708 / 252-2250$

$252-2361$

$708 / 202-2264$

$252-2361$

$708 / 252-2102$

$252-2361$

$708 / 252-2250$

252-2361

$708 / 252-2264$

$252-2361$

$708 / 252-2240$ 
WASTE MANAGEMENT

PROGRAM MANAGEMENT DIVISION:

Director: A. Creig Zook

$252-2361$

$708 / 252-2250$

ENVIRONMENTAL PROGRAMS BRANCH

Chief: Donna Green

$252-2361$

$708 / 252-2264$

ENVIRONMENTAL RESTORATION PROGRAM

Manager: Linda Freeman

$252-2361$

$708 / 252-2240$

WASTE MANAGEMENT PROGRAM

Manager: Evet Gonzalez

$252-2361$

$708 / 252-2276$

*DIAL AREA CODE

* RCRA/CERCLA/TSCA REGULATORY RESPONSE LEAD

$$
\mathrm{CH}-4
$$


ARGONNE NATIONAL LABORATORY (ANL EAST)

9700 SOUTH CASS AVENUE

ARGONNE, ILLINOIS 60439

FAX * COMMERCIAL

LABORATORY DIRECTOR: ALAN SCHREISHEIM

252-7923 708/252-3872

CHIEF OPERATIONS OFFICER: RONALD TEUNIS

252-2948 708/252-5569

\section{ENVIRONMENT}

ENVIRONMENT AND WASTE MANAGEMENT PROGRAMS

Director: Fred Brunner

$252-9642$

$708 / 252-3233$

ENVIRONMENTAL COMPLIANCE

Norbert Golchert

252-9642

$708 / 252-3912$

Julie Mathiesen

252-5965

$708 / 252-7128$

\section{ENVIRONMENTAL RESTORATION}

ENVIRONMENT AND WASTE MANAGEMENT PROGRAMS

Director: Fred Brunner

$252-9642$

$708 / 252-3233$

ENVIRONMENTAL PROJECTS

Jim Thuot

$252-9642$

$708 / 252-4911$

\section{WASTE MANAGEMENT}

ENVIRONMENT AND WASTE MANAGEMENT PROGRAMS

Director: Fred Brunner

252-7642

$708 / 252-3233$

WASTE MANAGEMENT

R. Max Schletter

$252-7190$

$708 / 252-2744$ 
ARGONNE AREA OFFICE (WEST) - DOE

ARGGNYE NATIONAL LABORATORY (WEST)

EBR 2 SITE, INEL

SCOVILLE, IDAHO 83415

P.O. BOX 2528

IDAHO FALLS, IDAHO 83403-2528

FAX* COMMERCIAL

DIRECTOF: ERNEST HUGHES

533-7422

200/533-7446

ENVIRONAEENT

Gregg Bass:*

$533-7422$

208/533-7184

ENVIRONMENTA1, RESTORATION

Greg Bass

533-7422 208/533-7184

WASTE MANAGEMENT

Greg Bass

533-7422 208/533-7184

* Dial area code

**RCRA/CERCLA/TSC.A REGULATORY RESPONSE LEAD

$\mathrm{CH}-6$ 
ARGONNE NATIONAL LABORATORY (ANL WEST)

INEL ARGONNE WEST SITE

IDAHO FALLS, IDAHO 83415

P.O. BOX 2528

IDAHO FALLS, IDAHO 83403

FAX * COMMERCIAL

DEPUTY ASSOCIATE LABORATORY DIRECTOR: JOHN SACKEIT

533-7249 208/533-7671

ENVIRONMENT

ENVIRONMENT, SAFETY \& WASTE MANAGEMENT

Manager: Gary Marshall

533-7344 208/533-7666

ENVIRONMENT \& W'ASTE MANAGEMENT

Manager: Paul Mikolaysic

533-7344 208/533-7163

ENVIRONMENTAL RESTORATION

ENVIRONMENT, SAFETY \& WASTE MANGEMENT

Manager: Gary Marshall

533-7344 208/533-7666

ENVIRONMENT \& WASTE MANAGEMENT

Manager: Paul Mikolaysic

533-7344 208/533-7163

\section{WASTE MANAGEMENT}

ENVIRONMENT, SAFETY \& WASTE MANAGEMENT

Manager: Gary Marshall

533-7344 208/533-7666

ENVIRONMENT \& WASTE MANAGEMENT

Manager: Paul Mikolaysic

533-7344 208/533-7163

* Dial area code

**RCRA/CERCLA/TSCA REGULATORY RESPONSE LEAD

$\mathrm{CH}-7$ 
BATAVIA AREA OFFICE - DOE

KIRK \& PINE STREET

BATAVIA, ILLINOIS 60510

P.O. BOX 2000

BATAVIA, ILLINOIS 60510

FAX*

840-3285

COMMERCIAL

AREA MANAGER: ANDREW MRAVCA

\section{ENVIRONMENT}

ENVIRONMENTAL COORDINATOR:

$$
\text { Jon Cooper } \star \star
$$

840-3285

$708 / 840-3281$

ENVIRONMENTAL RESTORATION

ENVIRONMENTAL COORDINATOR:

Jon Cooper

840-3285

$708 / 840-3281$

WASTE MANAGEMENT

ENVIRONMENTAL COORDINATOR:

$$
\text { Jon Cooper }
$$

840-3285

$708 / 840-3281$ 
FERMI NATIONAL ACCELERATOR LABORATORY (FERMILAB)

BATAVIA, ILLINOIS 60510

P.O. BOX 500

BATAVIA, ILLINOIS 60510

FAX *

840-2939

COMMERCIAL

DIRECTOR: JOHN PEOPLES

\section{ENVIRONMENT}

ASSOCIATE DIRECTOR FOR TECHNOLOGY

Dennis Theriot

ENVIRONMENTAL SAFETY \& HEALTH SECTION

Head: Don Cossairt ${ }^{\star \star}$

ENVIRONMENTAL PROTECTION GROUP

Leader: Don Cossairt (Acting)

840-3390

$708 / 840-3465$

\section{ENVIRONMENTAL RESTORATION}

ASSOCIATE DIRECTOR OF TECHNOLOGY

Dennis Theriot

840-2939

$708 / 840-3211$

ENVIRONMENTAL SAFETY \& HEALTH SECTION

Head: Don Cossairt

840-3390

$708 / 840-3465$

HEALTH \& SAFETY GROUP

Leader: Tim Miller

$840-3390$

$708 / 840-3019$

WASTE MANAGEMENT

ASSOCIATE DIRECTOR OF TECHNOLOGY

Dennis Theriot

840-2939

$708 / 840-3211$

ENVIRONMENTAL SAFETY \& HEALTH SECTION

Head: Don Cossairt

840-3390

$708 / 840-3465$

HEALTH \& SAFETY GROUP

Leader: Tim Miller
$840-3390$

$708 / 840-3019$

\section{* DIAL AREa CODE}

* RCRA/CERCLA/TSCA REGULATORY RESPONSE LEAD 
BROOKHAVEN AREA OFFICE - DOE

53 BELL AVENUE

BUILDING 464

UPTON, NEW YORK 11973

FAX * COMMERCIAL

AREA MANAGER: CARSON NEELY

282-3444 516/282-3424

\section{ENVIRONMENT}

ENGINEERING \& TECHNICAL MANAGEMENT BRANCH

Chief: Mike Butler**

282-3444

$516 / 282-3430$

ENVIRONMENT COMPLIANCE

Acting Manager: Caroline Polanish

282-3444

$516 / 282-5224$

\section{ENVIRONMENTAL RESTORATION}

ENGINEERING \& TECHNICAL MANAGEMENT BRANCH

Chief: Mike Butler

$282-3444 \quad 515 / 282-3430$

ENVIRONMENTAL RESTORATION

Program Manager: Gail Penny

$282-3444 \quad 516 / 282-3429$

WASTE MANAGEMENT

ENGINEERING \& TECHNICAL MANAGEMENT BRANCH

Chief: Mike Butler

282-3444

$515 / 282-3430$

WASTE OPERATIONS

Project Manager: Caroline Polanish

$282-3444 \quad 516 / 282-5224$

*DIAL AREA CODE

**RCRA/CERCLA/TSCA REGULATORY RESPONSE LEAD

$\mathrm{CH}-10$ 
BROOKHAVEN NATIONAL LABORATORY (BNL)

40 BROOKHAVEN AVENUE

UPTON, NEW YORK 11973

$282-5803$

DIRECTOR: NICHOLAS SAMIOS

\section{ENVIRONMENT}

REACTOR SAFETY \& SECURITY

Associate Director: M. Sue Davis

SAFETY \& ENVIRONMENTAL PROTECTION DIVISION

Head: Robert Casey

\section{ENVIRONMENTAL RESTORATION}

OFFICE OF ENVIRONMENTAL RESTORATION

Manager: Alan Raphael

\section{WASTE MANAGEMENT}

REACTOR SAFETY \& SECURITY

Associate Director: M. Sue Davis

SAFETY \& ENVIRONMENTAL SAFETY DIVISION

Head: Robert Casey

HAZARDOUS WASTE MANAGEMENT SECTION

Head: Leonard Emma
FAX*

$282-5584$

$516 / 282-4492$

282-7776

$516 / 282-5854$

$282-7618$

COMMERCIAL

$516 / 282-2772$

282-7618

$516 / 282-4654$

$282-5584 \quad 516 / 282-4492$

$282-7618 \quad 516 / 282-4654$

$516 / 666-3334$

* DIAL aRea code

**RCRA/CERCLA/TSCA REGULATORY RESPONSE LEAD

$$
\mathrm{CH}-11
$$


ENVIRONMENTAL MEASUREMENTS LABORATORY - DOE

376 HUDSON STREET

NEW YORK, NEW YORK 10014-3621

FAX * COMMERCIAL

LABORATORY DIRECTOR: PHILLIP KREY (ACTING)

$620-3600$

$212 / 620-3619$

ENVIRONMENT

ENVIRONMENTAL COMPLIANCE OFFICER

Herbert Feely* *

$620-3600 \quad 212 / 620-3627$

ENVIRONMENTAL RESTORATION

ENVIRONMENTAL COMPLIANCE OFFICER

Herbert Feely

$620-3600 \quad 212 / 620-3627$

WASTE MANAGEMENT

ENVIRONMENTAL COMPLIANCE OFFICER

Herbert Feely

$620-3600 \quad 212 / 620-3627$

*DIAL AREA CODE

*\#RCRA/CERCLA/TSCA REGULATORY RESPONSE LEAD

$\mathrm{CH}-12$ 
NATIONAL RENEWABLE ENERGY LABORATORY (NREL) AREA OFFICE - DOE 1617 COLE BOULEVARD

GOLDEN, COLORADO 80401

FAX

231-7394

AREA MANAGER: PAUL KEARNS

\section{ENVIRONMENT}

ESH/OPERATIONS DIVISION

Director: Jeffrey Baker**

ENVIRONMENTAL RESTORATION

ESH/OPERATIONS DIVISION

Director: Jeffrey Baker

\section{WASTE MANAGEMENT}

ESH/OPERATIONS DIVISION

Director: Jeffrey Baker

$$
\begin{array}{ll}
231-7394 & 303 / 231-1284 \\
231-7394 & 303 / 231-1284
\end{array}
$$

$231-7394$

$303 / 231-1284$

$231-7394 \quad 303 / 231-1284$

*DIAL AREA CODE

**RCRA/CERCLA/TSCA REgULATORY RESPONSE LEAD

$\mathrm{CH}-13$ 
NATIONAL RENEWABLE ENERGY LABORATORY (NREL - formerly SERI)

1617 COLE BOULEVARD

GOLDEN, COLORADO 80401

FAX*

COMMERCIAL

DIRECTOR: DWAYNE SUNDERMAN

231-1997

$303 / 231-7111$

\section{ENVIRONMENT}

SAFETY \& SECURITY OFFICE

Manager: Bill Griffing

$231-1381$

$303 / 231-1178$

\section{ENVIRONMENTAL RESTORATION}

SAFETY \& SECURITY OFFICE

Manager: Bill Griffing

$231-1381$

$303 / 231-1178$

WASTE MANAGEMENT

SAFETY \& SECURITY OFFICE

Manager: BIII Griffing

$231-1381$

$303 / 231-1178$ 
NEW BRUNSWICK LABORATORY - DOE

BUILDING 350

9800 SOUTH CASS AVENUE

ARGONNE, ILLINOIS 60439

FAX*

252-6256

COMMERCIAL

DIRECTOR: CARLETON BINGHAM

\section{ENVIRONMENT}

DEPUTY LABORATORY DIRECTOR

H. Rodney Martin

OPERATIONS SUPP. DIVISION

Director: Margaret Lachman**

\section{ENVIRONMENTAL RESTORATION}

DEPUTY LABORATORY DIRECTOR

H. Rodney Martin

OPERATIONS SUPP. DIVISION

Director: Margaret Lachman

252-6256

$708 / 252-2437$

$252-6256$

$708 / 252-2492$

$252-6256$

$708 / 252-2437$

$252-6256$

$708 / 252-2492$

\section{WASTE MANAGEMENT}

DEPUTY LABORATORY DIRECTOR

H. Rodney Martin

$252-6256$

$708 / 252-2437$

OPERATIONS SUPP. DIVISION

Director: Margaret Lachman
$252-6256 \quad 708 / 252-2492$ 
PRINCETON AREA OFFICE - DOE

PLASMA PHYSICS LABORATORY

U.S. ROUTE 1, NORTH

JAMES FORRESTAL CAMPUS

LOB BUILDING, ROOM B290

PRINCETON, NEW JERSEY 08542

P.O. BOX 102

PRINCETON, NEW JERSEY 08542-0102

FAX *

COMMERCIAL

AREA MANAGER: MILTON JOHNSON

243-2032

$609 / 243-3700$

\section{ENVIRONMENT}

ENVIRONMENTAL, SAFETY \& HEALTH BRANCH

Chief: Juris Balodiz

243-3777

$609 / 243-3709$

ENVIRONMENTAL ENGINEER

Allen Wrigley**

243-3777

$609 / 243-3710$

ENVIRONMENTAL RESTORATION

ENVIRONMENTAL, SAFETY \& HEALTH BRANCH

Chief: Juris Balodiz

243-3777

609/243-3709

ENVIRONMENTAL ENGINEER

Allen Wrigley

243-3777

$609 / 243-3710$

\section{WASTE MANAGEMENT}

ENVIRONMENTAL, SAFETY \& HEALTH BRANCH

Chief: Juris Balodiz

243-3777

609/243-3709

ENVIRONMENTAL ENGINEER

Allen Wigley

243-3777

$609 / 243-3710$

HEALTH PHYSICIST (RADIOACTIVE/MIXED WASTE)

Stephen Elwood

243-3777

$609 / 243-3715$

"DIAL AREA CODE

**RCRA/CERCLA/TSCA REGULATORY RESPONSE LEAD

$\mathrm{CH}-16$ 
PRINCETON UNIVERSITY PLASMA PHYSICS LABORATORY (PPPL)

JAMES FORRESTAL CAMPUS

U.S. ROUTE 1, NORTH

RECEIVING 3

PRINCETON, NEW JERSEY 08543

P.O. BOX 451

PRINCETON, NEW JERSEY 08543

FAX* COMMERCIAL

DIRECTOR: RONALD DAVIDSON

243-2749 609/243-3553

\section{ENVIRONMENT}

ES\&H/QA DEPARTMENT

Assoc. Director: John Delooper

243-2422

$609 / 243-3047$

ES\&H DIVISION

Head: Ellis Simon

243-2422

$609 / 243-2778$

Deputy Head: Joe Stencil

243-2525

$609 / 243-2529$

ENVIRONMENT \& HEALTH BRANCH

Head: Jerry Levine

243-2525

$609 / 243-3439$

\section{ENVIRONMENTAL RESTORATION}

OFFICE OF RESOURCE MANAGEMENT

Head: Ed Winkler

243-2749 609/243-2218

ER/WM ADMINISTRATION DIVISION

Head: Scott Larson

243-3366

$609 / 243-3387$

*DIAL AREA CODE

**RCAA/CERCLA/TSCA REGULATORY RESPONSE LEAD

$\mathrm{CH} \cdot 17$ 
WASTE MANAGEMENT

OFFICE OF RESOURCE MANAGEMENT

Head: Ed Winkler

243-2749

$609 / 243-2218$

ER/WM ADMINISTRATION DIVISION

Head: Scott Larson

243-3366

$609 / 243-3387$

WASTE MANAGEMENT BRANCH

Manager: Jim Scott**

243-3366

$609 / 243-3385$ 
Energy Technology Centers (ETC) 


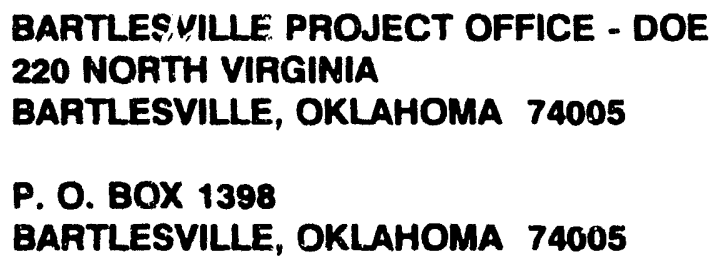

ENV:RONMENTAL RESTORATION

PROGRAM COORDINATOR-AEPT

Alex Crawley

$337-4418 \quad 918 / 237-4406$

ES\&H PROJECT MANAGER

David Alleman

$337-4418 \quad 918 / 337-4455$

\section{WASTE MANAGEMENT}

PROGRAM COORDINATOR-AEPT

Alex Crawley

$337-4418 \quad 918 / 337-4406$

ES\&H PROJECT MANAGEF

David Alleman

$337-4418 \quad 918 / 337-4455$ 
MORGANTOWN ENERGY TECHNOLOGY CENTER (METC) - DOE

3610 COLLINS FERRY ROAD

MORGANTOWN, WEST VIRGINIA 26507

P.O. BOX 880

MORGANTOWN, WEST VIRGINIA 26507-0880

DIRECTOR: THOMAS BECHTEL

FAX *

291-4292 COMMERCIAL

$304 / 291-4511$

ENVIRONMENT

OFFICE OF THE DIRECTOR

Chief Engineer: John Notestein

$291-4292$

$304 / 291-4232$

ENVIRONMENT

John Ganz *

$291-4292 \quad 304 / 291-4346$

\section{ENVIRONMENTAL RESTORATION}

OFFICE OF PROGRAM SUPPORT AND EVALUATION

Division Director: Jan Wachter

ENVIRONMENT

Joseph Martin

WASTE MANAGEMENT

OFFICE OF PROGRAM SUPPORT AND EVALUATION

Division Director: Jan Wachter

ENVIRONMENT

Jason Cook
$291-4403 / 69 \quad 304 / 291-4607$

$291-4403 / 69 \quad 304 / 291-4607$

$291-4403 / 69 \quad 304 / 291-4447$

$291-4403 / 69 \quad 304 / 291-4718$ 
EG \& G WASC, INC - MORGANTOWN

3610 COLLINS FERRY ROAD

MORGANTOWN, WEST VIRGINIA 26505

P.O. BOX 880

MURGANTOWN, WEST VIRGINIA 26505

FAX* COMMERCIAL

MANAGER: GARY MILLAR

291-4035

304/291-4539

\section{ENVIRONMENT}

CUSTOMER SERVICE MANAGER

Randall Wotring

$291-4403 / 69 \quad 304 / 291-4298$

ENVIRONMENTAL SUPPORT TEAM

Carmen Romano

$291-4403 / 69 \quad 304 / 291-4214$

\section{ENVIRONMENTAL RESTORATION}

CUSTOMER SERVICE MANAGER

Randall Wotring

$291-4403 / 69 \quad 304 / 291-4298$

ENVIRONMENTAL SUPPORT TEAM

Carmen Romano

$291-4403 / 69 \quad 304 / 291-4214$

WASTE MANAGEMENT

CUSTOMER SERVICE MANAGER

Randall Wotring

$291-4403 / 69 \quad 304 / 291-4298$

ENVIRONMENTAL SUPPORT TEAM

Carmen Romano

$291-4403 / 69 \quad 304 / 291-4214$

**RCRA/CERCLA/TSCA REGULATORY RESPONSE LEAD

$$
\text { ETC - } 3
$$




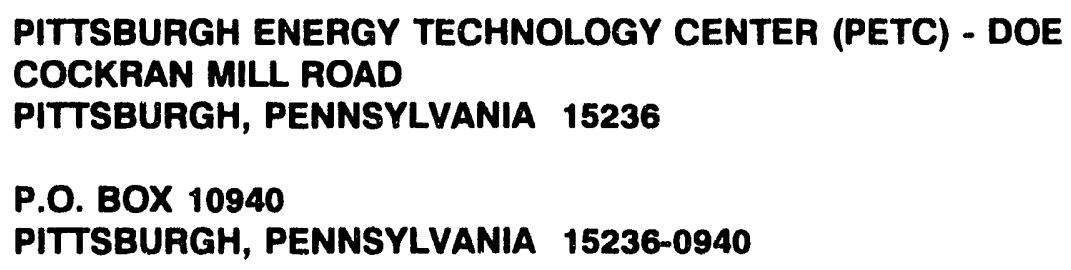

\begin{tabular}{lcc} 
& FAX & COMMERCIAL \\
\hline DIRECTOR: SUN CHUN & $892-6127$ & $412 / 892-6122$
\end{tabular}

\section{ENVIRONMENT}

OFFICE OF ADMINISTRATION

Associate Director: Melvin Keller

$892-6216 \quad 412 / 892-5772$

ENGINEERING, ENVIRONMENT, HEALTH \& SAFETY DIVISION

Director: George Bellas

$892-6228 \quad 412 / 892-6184$

ENVIRONMENTAL BRANCH

Supervisor: Elias George ${ }^{\star \star}$

$892-6228-412 / 892-4497$

\section{ENVIRONMENTAL RESTORATION}

OFFICE OF ADMINISTRATION

Associate Director: Melvin Keller

892-6216

$412 / 892-5772$

ENGINEERING, ENVIRONMENT, HEALTH \& SAFETY DIVISION

Director: George Bellas

892-6228

$412 / 892-6184$

ENVIRONMENTAL BRANCH

Supervisor: Elias George

$892-6228$

$412 / 892-4497$ 
WASTE MANAGEMENT

OFFICE OF ADMINISTRATION

Associate Director: Melvin Keller

892-6216

$412 / 892-5772$

ENGINEERING, ENVIRONMENT, HEALTH \& SAFETY DIVISION

Director: George Bellas

892-6228

$412 / 892-6184$

ENVIRONMENTAL BRANCH

Supervisor: Elias George

892-6228

$412 / 892-4497$

*DIAL AREA CODE

**RCRA/CERCLA/TSCA REGULATORY RESPONSE LEAD

ETC - 5 
Fernald Field Office (FN) 


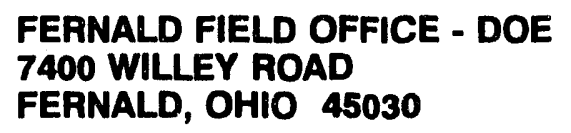

\section{ENVIRONMENT}

TECHNICAL SUPPORT

Assistant Manager: Walter Quaider**

ENVIRONMENTAL COMPLIANCE DIVISION

Director: Vacant

\section{ENVIRONMENTAL RESTORATION}

ENVIRONMENTAL RESTORATION

Assistant Manager: Jack Craig

LOW LEVEL WASTE STORAGE UNIT DIVISION

Director: Vacant

FACILITIES AND ENVIRONMENTAL MEDIA DIVISION

Director: Vacant

\section{WASTE MANAGEMENT}

SITE OPERATIONS

Assistant Manager: Ray Hansen (Acting)

WASTE MANAGEMENT DIVISION

Director: Vacant

D \& D, SAFE SHUTDOWN \& SITE SERVICES DIVISION

Director: Vacant

\section{*DIAL AREA CODE}

**RCRA/CERCLA/TSCA REGULATORY RESPONSE LEAD 
WESTINGHOUSE ENVIRONMENTAL MANAGEMENT COMPANY OF OHIO

FERNALD

7400 WILLEY ROAD

FERNALD, OHIO 45030

P.O. BOX 398704

CINCINNATI, OHIO 45239-8704

FAX*

$738-8976$

PRESIDENT: NICK KAUFMAN

ENVIRONMENT

ENVIRONMENTAL COMPLIANCE \& QUALITY ASSURANCE

Manager: Don Dubois

SITE-WIDE COMPLIANCE

Manager: Steve Beckman

ENVIRONMENTAL RESTORATION

ENVIRONMENTAL RESTORATION

Manager: Jim Theising

\section{WASTE MANAGEMENT}

ENVIRONMENTAL MANAGEMENT

Staff Manager: Don Paine

WASTE MANAGEMENT PROGRAM

Program Manager: Jim Long
$738-6236$

$513 / 738-8682$

$738-6236$

$513 / 738-9228$

$738-8976$

$513 / 738-9230$

$738-8428$

$513 / 738-9231$

$738-8978$

$513 / 738-8450$

*DIAL AREA CODE

**RCRA/CERCLA/TSCA REGULATORY RESPONSE LEAD

FN - 2 
Idaho Field Office (ID) 
IDAHO FIELD OFFICE - DOE

785 DOE PLACE

IDAHO FALLS, IDAHO 83401-1562

FAX*

526-5406

MANAGER: AUGUSTINE PITROLO

\section{ENVIRONMENT}

OFFICE OF ENVIRONMENT, SAFETY \& HEALTH OVERSIGHT

Manager: Gerald Bowman

ENVIRONMENTAL \& QUALITY ASSURANCE DIVISION

Director: Margaret Fisher

ENVIRONMENTAL COMPLIANCE BRANCH

Chief: Charles Ljungberg

SITE ENGINEERING \& SUPPORT

Assistant Manager: George Dials

ENVIRONMENTAL SUPPORT DIVISION

Director: Margaret Hinman**

\section{ENVIRONMENTAL RESTORATION}

ENVIRONMENTAL RESTORATION \& WASTE MANAGEMENT

Assistant Manager: Thomas Burns, Jr.

ENVIRONMENTAL RESTORATION DIVISION

Director: Alice Williams

BURIED WASTE PROGRAM

Manager: Donald MacDonald

REMEDIATION PROGRAM

Manager: Lisa Green *
526-1184

208/526-7653

$526-1926$

$526-1926$

$526-1926$

$526-1184$

$526-1184$

208/526-0657

526-1184

208/526-0972

526-1184 208/526-6520

$526-1184$

208/526-0417

*DIAL AREA CODE

* RCRA/CERCLA/TSCA REGULATORY RESPONSE LEAD

ID - 1 
ENVIRONMENTAL RESTORATION AND WASTE MANAGEMENT

Assistant Manager: Thomas Burns, Jr.

WASTE MANAGEMENT DIVISION

Director: Walter Sato

526-1184

208/526-0193

NLLW PROGRAM

Manager: Ron Sandwina

526-1184

$208 / 526-0920$

WASTE OPERATIONS

Manager: Joel Case

526-1184

208/526-5558

ADVANCED SYSTEMS

Manager: Geoff Beausoleil

526-1184

208/526-5558

ID - 2 


\section{BABCOCK AND WILCOX/INEL \\ 1580 SAWTELLE, MS-2700 \\ IDAHO FALLS, IDAHO 83403}

P.O. BOX 1469, MS-2700

IDAHO FALLS, IDAHO 83403

FAX*

COMMERCIAL

PRESIDENT \& GENERAL MANAGER:

Greg Baker

$526-1646 \quad 208 / 526-6755$

\section{ENVIRONMENT}

COMPLIANCE, ASSESSMENT \& ENVIRONMENT

Director: Arvid Jensen

$526-6361 \quad 208 / 526-6746$

ENVIRONMENTAL PROTECTION

Manager: David Blumberg

$526-6077 \quad 208 / 526-6505$

\section{ENVIRONMENTAL RESTORATION}

COMPLIANCE, ASSESSMENT \& ENVIRONMENT

Director: Arvid Jensen

$526-6361 \quad 208 / 526-6746$

ENVIRONMENTAL PROTECTION

Manager: David Blumberg

526-6077 208/526-6505

ENVIRONMENT, SAFETY, HEALTH \& WASTE MANAGEMENT

Director: Larrie Trent

526-1646 208/526-9132

WASTE MINIMIZATION COORDINATOR

Kris Klossner

526-3417 208/526-6013

WASTE OPERATIONS

Manager: Robert Moss

526-1646 208/526-6513 
WASTE MANAGEMENT

ENVIRONMENT, SAFETY, HEALTH \& WASTE MANAGEMENT

Director: Larrie Trent

526-1646

208/526-9132

WASTE MINIMIZATION COORDINATOR

Kris Klossner

526-3417

208/526-6013

WASTE OPERATIONS

Manager: Robert Moss

208/526-6513 
EG \& G IDAHO, INC.

765 LNDSAY BOULEVARD

IDAHO FALLS, IDAHO 83415-2420

P.O. BOX 1625

IDAHO FALLS, IDAHO 83415-2420

FAX * COMMERCIAL

GENERAL MANAGER: JAMES OKESON

526-8339 208/526-1969

ENVIRONMENT

ES \& Q DEPARTMENT

Manager: Richard Watkins

526-5008 208/526-6886

ENVIRONMENTAL PROGRAMS GROUP

Manager: Beth Reyes

526-1998 208/526-1651

ENVIRONMENTAL TECHNICAL SUPPORT

David Hutchinson

526-9779 208/526-9263

ENVIRONMENTAL RESTORATION AND WASTE

MANACEMENT

ENVIRONMENTAL RESTORATION DEPARTMENT

Manager: James McAnally

526-6902 208/526-2835

PROGRAM SUPPORT GROUP

Manager: Susan Stiger

526-8632 208/526-0457

ID - 5 
WESTINGH ZUSE IDAHO NUCLEAR COMPANY, INC.

1 DS5 FREMONT DRIVE

IDAHO FALLS, IDAHO 83415

P.O. BOX 4000

IDAHO FALLS, IDAHO 83415

FAX* COMMERCIAL

PRESIDENT: WILLIAM MOFF:TT

526-4550 208/526-0998

\section{ENVIRONMENT}

ENVIRONMENTAL SAFETY \& HEALTH DEPARTMENT

V.P. \& MEngger: Anthony Umek

526-9792 208/526-9690

ENVIRONMENTAL ASSURANCE

Manager: Tíomas Pointer

526-5945 208/526-4432

ENVIRONMENTAL PERIMITTING \& REGULATIONS

Manager: Gary Franz

526-0665 208/526-8560

ENVIRONMENTAL PERMITTING

Manager: Deborah Wiggins

526-0665 208/526-9989

RCRA COMPLIANCE

Manager: Steve Birrer

$526-0665 \quad 208 / 526-3427$

ENVIRONMENTAL ASSESSMENT

Manager: Jim Linhart

$526-0665 \quad 208 / 526-8910$

*DIAL AREA CODE

**RCRA/CERCLA/TSCA REGULATORY RESPONSE LEAD

ID -6 
ENVIRONMENTAL RESTORATION

ENVIRONMENTAL SAFETY \& HEALTH DEPARTMENT

V.P. \& Manager: Anthony Umek

526-9792 208/526-9690

ENVIRONMENTAL RESTORATION

Manager: 0 . Kenner Earle

526-0665 208/526-1114

SITE REMEDIATION

Manager: Dee Williamson

526-5945 208/526-5916

RESTORATION PROGRAM AND ADMINISTRATION

Manager: John Coody

526-0665 208/526-0633

ENVIRONMENTAL CHARACTERIZATION

Manager: Phillip Peistrup

526-0665 208/526-4207

\section{WASTE MANAGEMENT}

ENVIRONMENTAL SAFETY \& HEALTH DEPARTMENT

V.P. \& Manager: Anthony Umek

526-9792 208/526-9690

ENVIRONMENTAL PERMITTING AND REGULATIONS

Manager: Gary Franz

$526-0665 \quad 208 / 526-8560$

RCRA COMPLIANCE

Manager: Steve Birrer

526-0665 208/526-3427

ENVIRONMENTAL PERMITTING

Manager: Deborah Wiggins

526-0665 208/526-9989

*DIAL AREA CODE

**RCRA/CERCLA/TSCA REGULATORY RESPONSE LEAD

ID -7 
WEST VALLEY DEMONSTRATION PROJECT OFFICE - DOE

10300 ROCK SPRINGS ROAD

WEST VALLEY, NEW YORK 14171-0191

FAX*

COMMERCIAL

DIRECTOR: THOMAS ROWLAND

$942-4703$

$716 / 942-4312$

\section{ENVIRONMENT}

REGULATORY COMPLIANCE OFFICE

Manager: Richard Provencher**

$942-4703$

$716 / 942-4101$

Elizabeth Matthews

942-4703

$716 / 942-4930$

ENVIRONMENTAL RESTORATION

REGULATORY COMPLIANCE OFFICE

Manager: Richard Provencher

942-4703

716/942-4101

\section{WASTE MANAGEMENT}

TECHNOLOGY PROGRAM OFFICE

Manager: Steve Ketola

$942-4703$

$716 / 942-4314$

* DIAL AREA CODE

*\#CRA/CERCLA/TSCA REGULATORY RESPONSE LEAD

ID - 8 
Naval Petroleum Reserve (NOSR) 
NAVAL PETROLEUM RESERVE (CALIFORNIA) - DOE

28590 HIGHWAY 119

TUPMAN, CALIFORNIA 93276

P.O. BOX 11

TUPMAN, CALIFORNIA 93276

FAX * COMMERCIAL

DIRECTOR: DANNY HOGAN

763-6699

805/763-6011

ENVIRONMENT

PLANNING, ANALYSIS \& PROGRAM SUPPORT DIVISION

Director: Jim Killen

763-6699 805/763-6038

Environmental Protection Specialist: Gary Walker**

$763-6699 \quad 805 / 763-6021$

\section{ENVIRONMENTAL RESTORATION}

PLANNING, ANALYSIS \& PROGRAM SUPPORT DIVISION

Director: Jim Killen

763-6699

$805 / 763-6038$

WASTE MANAGEMENT

PLANNING, ANALYSIS \& PROGRAM SUPPORT DIVISION

Director: Jim Killen

$763-6699$

805/763-6038

*DIAL AREA CODE

**RCRA/CERCLA/TSCA REGULATORY RESPONSE LEAD

NOSR - 1 
BECHTEL PETROLEUM OPERATION, INC.

28590 HIGHWAY 119

TUPMAN, CALIFORNIA 93216

P.O. BOX 127

TUPMAN, CALIFORNIA 93276

FAX* COMMERCIAL

GENERAL MANAGER: JIM WATSON

765-5365

805/763-6202

ENVIRONMENT

ENVIRONMENTAL SERVICES

Manager: Richard Donahoe

$763-6171$

$805 / 763-6573$

ENVIRONMENTAL RESTORATION

ENVIRONMENTAL SERVICES

Manager: Richard Donahoe

$763-6171 \quad 805 / 763-6573$

WASTE MANAGEMENT

ENVIRONMENTAL SERVICES

Manager: Richard Donahoe

$763-6171$

$805 / 763-6573$

*\#RCRA/CERCLA/TSCA REGULATORY RESPONSE LEAD

NOSR - 2 
NAVAL PETROLEUM \& OIL SHALE RESERVE (WYOMING, COLORADO, \& UTAH) - DOE 907 NORTH POPLAR STREET

SUITE 150

CASPER, WY 82601

FAX* COMMERCIAL

DIRECTOR: C. RAY WILLIAMS

$261-5817 \quad 307 / 261-5161$

\section{ENVIRONMENT}

David Miles**

261-5817 $307 / 261-5071$

\section{ENVIRONMENTAL RESTORATION}

David Miles

$261-5817$

$307 / 261-5071$

WASTE MANAGEMENT

David Miles

261-5817 $307 / 261-5071$ 
Nevada Field Office (NV) 
NEVADA FIELD OFFICE - DOE

2753 SOUTH HIGHLAND AVENUE

LAS VEGAS, NEVADA 89109

P.O. BOX 98518

LAS VEGAS, NEVADA 89193-8518

FAX* COMMERCIAL

MANAGER: NICK AQUILINA

295-1876

$702 / 295-3211$

\section{ENVIRONMENT}

OFFICE OF ASSISTANT MANAGER FOR ENVIRONMENTAL

SAFETY, SECURITY \& HEALTH

Assistant Manager: Bruce Church

295-1062

$702 / 295-3201$

ENVIRONMENTAL PROTECTION DIVISION

Director: Donald Elle**

295-0838

$702 / 295-1146$

\section{ENVIRONMENTAL RESTORATION}

OFFICE OF ASSISTANT MANAGER FOR ENVIRONMENTAL RESTORATION AND WASTE MANAGEMENT

Assistant Manager: Joseph Fiore (Acting)

295-1810

$702 / 295-7063$

ENVIRONMENTAL RESTORATION \& WASTE MANAGEMENT DIVISION

Director: Stephen Mellington (Acting)

$295-1810 \quad 702 / 295-0409$

\section{WASTE MANAGEMENT}

OFFICE OF ASSISTANT MANAGER FOR ERWM

Assistant Manager: Joseph Fiore (Acting)

295-1810

702/295-7063

WASTE MANAGEMENT DIVISION

Director: Layton O'Neill

295-1810

$702 / 295-3181$

*DIAL AREA CODE

**RCRA/CERCLA/TSCA REGULATORY RESPONSE LEAD

NV -1 
EG \& G ENERGY MEASUREMENTS INC.

316 EAST ATLAS CIRCLE

NORTH LAS VEGAS, NEVADA 89030

P.O. BOX 1912

LAS VEGAS, NEVADA 89125

FAX * COMMERCIAL

GENERAL MANAGER: PETER ZAVATTARO

295-0562 702/295-5511

\section{ENVIRONMENT}

ASSISTANT GENERAL MANAGER FOR PERFORMANCE

ASSURANCE

Thomas Edwards

295-0562

295-3109

$702 / 295-0442$

ES \& H DIVISION

Director: Jackson Maddox

$295-5229$

$702 / 295-2955$

ENVIRONMENTAL COMPLIANCE OFFICE

Section Head: James Kessler

295-5229

$702 / 295-2960$

\section{ENVIRONMENTAL RESTORATION}

ASSISTANT GENERAL MANAGER FOR PERFORMANCE ASSURANCE

Thomas Edwards

295-0562

$702 / 295-0442$

ES \& H DIVISION

Director: Jackson Maddox

295-5229 702/295-2955

ENVIRONMENTAL COMPLIANCE OFFICE

Section Head: James Kessler

$295-5229$

$702 / 295-2960$

*DIAL AREA CODE

**RCRA/CERCLA/TSCA REGULATORY RESPONSE LEAD

NV -2 


\section{WASTE MANAGEMENT}

ASSISTANT GENERAL MANAGER FOR PERFORMANCE ASSURANCE

Thomas Edwards

295-0562

295-3109

ES \& H DIVISION

Director: Jackson Maddox

ENVIRONMENTAL COMPLIANCE OFFICE

Section Head: James Kessler
$295-5229$

$702 / 295-2955$

$295-5229$

$702 / 295-2960$

$$
\text { NV - } 3
$$




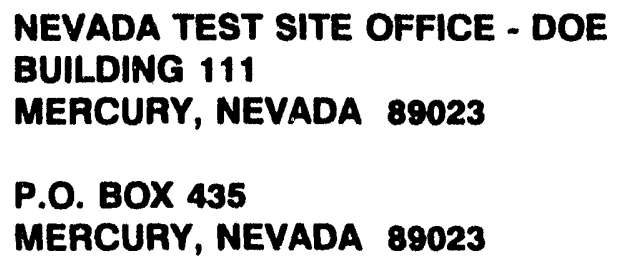

DIRECTOR: JOHN STEIIART

\section{ENVIRONMENTAL RESTORATION}

ENVIRONMENTAL RESTORATION AND WASTE MANAGEMENT DIVISION

Assistant Manager: Joseph Fiore (Acting)

ENVIRONMENTAL RESTORATION DIVISION

Director: Stephen Mellington (Acting) ${ }^{\star \star}$

\section{WASTE MANAGEMENT}

WASTE MANAGEMENT DIVISION

Chief: Layton O'Neill

TECHNOLOGY DEVELOPMENT AND PROGRAM MANAGEMENT DIVISION

Director: John Hall (Acting) 
REYNOLDS ELECTRICAL \& ENGINEERING CO., INC.

2501 WYANDOTTE STREET

LAS VEGAS, NEVADA 89102

P.O. BOX 98521

LAS VEGAS, NEVADA 89193-8521

FAX

GENERAL MANAGER: DALE FRASER

295-2361 702/295-3379

\section{ENVIRONMENT}

ENVIRONMENT, SAFETY \& HEALTH DIVISION

Manager: Anson Burlingame

295-7699

$702 / 295-7785$

HEALTH PROTECTION DEPARTMENT

Manager: Harrison Kerschner

295-7699 702/295-6212

ENVIRONMENTAL COMPLIANCE OFFICE:

Environmental Compliance Officer: Frank Bingham

295-7233 702/295-7221

ENVIRONMENTAL REVIEW \& ASSISTANCE SECTION

Orin Haworth

295-7233 702/295-7374

ENVIRONMENTAL ASSESSMENT SECTION

Tom Taylor

295-7233 $\quad 702 / 295-7947$

\section{ENVIRONMENTAL RESTORATION}

ENVIRONMENT, SAFETY \& HEALTH DIVISION

Manager: Howard Dickson

295-2059 702/295-7145

ENVIRONMENTAL RESTORATION AND TECHNOLOGY
DEVELOPMENT DEPARTMENT

Manager: Robert Dodge

295-1420

$702 / 295-1632$

*DIAL AREA CODE

**RCRA/CERCLA/TSCA REGULATORY RESPONSE LEAD

NV -5 
WASTE MANAGEMENT

ENVIRONMENT, SAFETY \& HEALTH DIVISION

Manager: Howard Dickson

295-2059 702/295-7145

WASTE OPERATIONS DEPARTMENT

Manager: Gene Kendall

$295-1420 \quad 702 / 295-5888$

ANALYTICAL SERVICES DEPARTMENT

Manager: Lee Sygitowicz

295-4773 702/295-6204

*DIAL AREA CODE

**RCRA/CERCLA/TSCA REGIILATORY RESPCASEE LEÁ

NV -6 
PACIFIC AREA SUPPORT OFFICE - DOE

HICKAM AIR FORCE BASE

BUILDING T-3225

HONOLULU, HAWAII 96820

P.O. BOX 29939

HONOLULU, HAWAII 96820

FAX* COMMERCIAL

PACIFIC AREA SUPPORT OFFICE: GRACE PLUMMER

422-9217 808/422-9211

OFFICE OF ASSISTANT MANAGER FOR TECHNICAL SUPPORT (NV)

ASSISTANT PROGRÁM MANAGER: DORNER SCHUELLER

702-295-1545

\section{ENVIRONMENT}

DEPUTY ASSISTANT SECRETARY FOR HEALTH (EH-40)

Dr. Harry Pettengill

903-3445 301/903-7030

OFFICE OF HEALTH PHYSICS/INDUSTRIAL HYGIENE PROGRAMS

Director: C. Rick Jones

903-7773 301/903-6061

HEALTH PHYSIC NROGRAMS DIVISION

Director: R. Thomas Bell

903-7773 $\quad 301 / 903-3889$

SITE REPRESENTATIVE

Bill Jackson

$422-9217$

$808 / 422-9203$

\section{ENVIRONMENTAL RESTORATION}

ENVIRONMENTAL RESTORATION \& WASTE MANAGEMENT DIVISION

Assistant Manager: Joseph Fiore (Acting)

$295-5300 \quad 702 / 295-7063$

ENVIRONMENTAL RESTORATION BRANCH

Director: Stephen Mellington (Acting) ${ }^{\star \star}$

$295-1810 \quad 702 / 295-0409$

*DIAL AREA CODE

**RCRA/CERCLA/TSCA REGULATORY RESPONSE LEAD

NV - 7 
WASTE MANAGEMENT

ENVIRONMENTAL RESTORATION \& WASTE MANAGEMENT DIVISION

Assistant Manager: Joseph Fiore (Acting)

$702 / 295-7063$

WASTER MANAGEMENT DIVISION

Chief: Layton O'Neill

$295-1810$

702/295-0996

*DIAL AREA CODE

**RCRA/CERCLA/TSCA REGULATORY RESPONSE LEAD

NV - 8 
Oak Ridge Field Office (OR) 
OAK RIDGE FIELD OFFICE - DOE

200 ADMINISTRATION ROAD

OAK RIDGE, TENNESSEE 37831

P.O. BOX 2001

OAK RIDGE, TENNESSEE 37831

\begin{tabular}{lcc} 
& FAX & COMMERCIAL \\
\hline MANAGER: JOE LAGRONE & $576-0006$ & $615 / 576-4444$
\end{tabular}

\section{ENVIRONMENT}

OFFICE OF ASSISTANT MANAGER FOR ENVIRONMENT, SAFETY \& QUALITY

Assistant Manager: Rodney Nelson

576-9686 615/576-0891

Deputy Assistant Manager: H. Wayne Hibbitts

$576-9686 \quad 615 / 576-0893$

ENVIRONMENTAL PROTECTION DIVISION

Director: Peter Gross

$576-0746 \quad 615 / 576-0845$

ENVIRONMENTAL OPERATIONS BRANCH

Chief: David Allen

$576-0746 \quad 615 / 576-0411$

ENVIRONMENTAL REMEDIATION BRANCH

Chief: Margaret Wilson**

$576-0746 \quad 615 / 576-8528$

\section{ENVIRONMENTAL RESTORATION}

OFFICE OF ASSISTANT MANAGER FOR ENVIRONMENTAL

RESTORATION \& WASTE MANAGEMENT

Assistant Manager: William Adams

576-3770 615/576-0742

Deputy Assistant Manager: Bryan Walker

576-3770 615/576-0716

ENVIRONMENTAL RESTORATION DIVISION

Director: Robert Sleeman

$576-7042 \quad 615 / 576-0715$ 
OAK RIDGE REMEDIATION BRANCH

Chief: W. Nelson Lingle

$576-7042$

$615 / 576-5580$

RESTORATION PROGRAM INTEGRATION BRANCH

Chief: Clayton Bist

$576-7042$

$615 / 576-6821$

WELDON SPRING SITE OFFICE

Site Manager: Stephen McCracken

477-0739

$314 / 441-8978$

FORMERLY UTILIZED SITES REMEDIATION ACTION PROGRAM

Director: Lester Price

\section{WASTE MANAGEMENT}

WASTE MANAGEMENT

Director: Larry Radcliffe

$576-5333$

$615 / 576-9212$

WASTE OPERATIONS \& TECHNOLOGY DEVELOPMENT BRANCH

Chief: Douglas Underwood

$576-5333$

$615 / 576-0728$

WASTE OPERATIONS \& TECHNOLOGY DEVELOPMENT BRANCH

Chief: Marianne Heiskell

$576-5333$

$615 / 576-0314$

K-25 SITE OFFICE

Site Manager: Tom Tison

$576-4724$

$615 / 576-2990$

*DIAL AREA CODE

* RCRA/CERCLA/TSCA REGULATORY RESPONSE LEAD

OR - 2 
MARTIN MARIETTA ENERGY SYSTEMS, INC. (MMES)

CENTRAL STAFF OFFICES

HIGHWAY 58 BLAIR ROAD

BUILDING K-100\%, MS 7169

OAK RIDGE, TENNESSEE 37831-7169

P.O. BOX 2003, BUILDING K-1001, MS 7169

OAK RIDGE, TENNESSEE $37830-7169$

FAX*

COMMERCIAL

ENVIRONMENT

COMPLIANCE, EVALUATIONS \& POLICY

Vice President: Fred Mynatt (BLDG. K1001, MS-7169)

$576-7536$

$615 / 574-4182$

ENVIRONMENTAL COMPLIANCE

Director: Michael Mitchell (BLDG. K1001, MS 7155)

Deputy Director: Conrad Stair (BLDG. K1001, MS 7155)

576-6706 615/576-8006

576-6706 615/576-5586

NEPA ENVIRONMENTAL MONITORING \& RESOURCE MANAGEMENT PROGRAM

Manager: Jim Rogers (BLDG. K1001, MS 7155)

$576-6714 \quad 615 / 574-8982$

CAA COMPLIANCE PROGRAM

Manager: Rodney Kingrea (BLDG. K1001, MS 7155)

$576-6714 \quad 615 / 576-6210$

TSCA, RCRA COMPLIANCE PROGRAM

Manager: Tom Perry (BLDG. K1001, MS 7155)

$576-6706 \quad 615 / 576-5587$

CWA COMPLIANCE PROGRAM

Manager: Sara Welch (BLDG. K1001, MS 7155)

576-6706 615/574-7864

STATE OVERSIGHT PROGRAM

Manager: Terry Cothron (BLDG. K1001, MS 7155)

$576-6714 \quad 615 / 576-5283$

CERCLA, SERA \& GROUNDWATER PROGRAM

Manager: Mike Ambrose (BLDG. K1001, MS 7155) $\quad 576-6714 \quad 615 / 576-6213$

*DIAL AREA CODE

**RCRA/CERCLA/TSCA REGULATORY RESPONSE LEAD

OR - 3 


\section{ENVIRONMENTAL RESTORATION}

ENVIRONMENTAL RESTORATION AND WASTE MANAGEMENT PROGRAMS

Vice President: J. Robert Merriman (Located at K-25 Site)

Deputy Manager: Finnis Patton (BLDG. K1001, MS 7169)

574-9936

$615 / 576-5800$

Special Assistant: Lanny Bates (BLDG K1001, MS 7169)

$576-4014$

$615 / 574-2991$

$576-4014$

$615 / 574-8995$

ENVIRONMENTAL RESTORATION DIVISION

Director: Fran DeLozier (BLDG. K1330, MS 7298)

$574-9538$

$615 / 576-3712$

K-25 ENYIRONMENTAL RESTORATION PROGRAM OFFICE

Manager: Ken Cook (BLDG. K1330, MS 7298)

$574-9538$

$615 / 574-9352$

Y-12 ENVIRONMENTAL RESTORATION PROGRAM OFFICE

Manager: William Brown (BLDG. 9983-AG, MS 8247)

$576-3808$

$615 / 576-5706$

ORNL ENVIRONMENTAL RESTORATION PROGRAM OFFICE

Manager: Bruce Kimmel (BLDG. 7078-A, MS 6402)

574-5969

$615 / 576-0311$

PADUCAH ENVIRONMENTAL RESTORATION PROGRAM OFFICE

Manager: Dennis Cope

$441-6049$

$502 / 441-6126$

PORTSMOUTH ENVIRONMENTAL RESTORATION PROGRAM OFFICE

Manager: T. David Taylor

$897-3752$

$614 / 897-2322$

*DIAL AREA CODE

**RCRA/CERCLA/TSCA REGULATORY RESPONSE LEAD

OR -4 


\section{WASTE MANAGEMENT}

CENTRAL WASTE MANAGEMENT DIVISION

Director: Charles Frye (BLDG. K1037, MS 7357)

576-6222 $\quad 615 / 574-9999$

PROGRAM MANAGEMENT

Deputy Director: Paul Franco

576-6222 615/574-0146

TECHNICAL PROGRAMS

Deputy Director: Lance Mezga

$576-6222$

$615 / 574-7258$

Mixed Waste Treatment Fasility Manager: Bob Hightower

$576-6222$

$615 / 574-6777$

*DIAL AREA CODE

**RCRA/CERCLA/TSCA REGULATORY RESPONSE LEAD

$$
\text { OR - } 5
$$


OAK RIDGE NATIONAL LABORATORY SITE OFFICE - DOE

BETHEL VALLEY ROAD

BLDG. $4500 \mathrm{~N}$, ROOM 215

OAK RIDGE, TENNESSEE, 37831-8610

P.O. BOX 2001

OAK RIDGE, TENNESSEE $37831-8610$

FAX*

$576-4511$

$574-9275$

$574-9275$

$615 / 576-1952$

Chief: Connor Matthews ${ }^{\star \star}$

\section{ENVIRONMENTAL RESTORATION}

ENERGY PROGRAMS DIVISION

Director: Thomas Jelinek

LABORATORY OPERATIONS BRANCH

Chief: Connor Matthews

WASTE MANAGEMENT

ENERGY PROGRAMS DIVISION

Director: Thomas Jelinek

LABORATORY OPERATIONS BRANCH

Chief: Connor Matthews
574-9275

$615 / 576-1952$

$574-9275 \quad 615 / 576-4876$

$574-9275$

$615 / 576-1952$
574-9275 $\quad 615 / 576-4876$

*DIAL AREA CODE

**RCRA/CERCLA/TSCA REGULATORY RESPONSE LEAD

OR - 6 
OAK RIDGE NATIONAL LABORATORY (ORNL) - MMES

1 BETHEL VALLEY ROAD

BUILDING 4500 N, ROOM 215

OAK RIDGE, TENNESSEE 37831

P.O. BOX 2008

OAK RIDGE, TENNESSEE $37831-6255$

FAX * COMMERCIAL

DIRECTOR: ALVIN TRIVELPIECE

576-2912

$615 / 576-2900$

\section{ENVIRONMENT}

ENVIRONMENTAL, SAFETY AND HEALTH COMPLIANCE

Director: Jerry Swanks

$576-8739 \quad 615 / 574-6688$

OFFICE OF ENVIRONMENTAL COMPLIANCE AND

DOCUMENTION

Director: Frank Kornegay

$576-6616 \quad 615 / 574-5776$

ENVIRONMENTAL REVIEW AND DOCUMENTION SECTION

Head: Helen Braunstein

$576-6537$

$615 / 574-5774$

ENVIRONMENTAL COMPLIANCE SECTION

Head: Carroll Nix

$576-6196 \quad 615 / 574-7363$

\section{ENVIRONMENTAL RESTORATION}

ENVIRONMENTAL RESTORATION AND WASTE MANAGEMENT PROGRAMS

Vice President: J. Robert Merriman (Located at K-25 Site)

574-9936 615/576-5800

Deputy Manager: Finnis Patton (Bldg 1001, MS 7169)

574-4014 615/574-2991

Special Assistant: Lanny Bates (Bldg ijo1, MS 7169)

$574-4014$

615/574-8995

ENVIRONMENTAL RESTORATION DIVISION

Director: Fran DeLozier (BIdg 1330, MS 7298)

$574-9538$

$615 / 576-3712$

*DIAL AREA CODE

**RCRA/CERCLAVTSCA REGULATORY RESPONSE LEAD

OR - 7 
ORNL ENVIRONMENTAL RESTORATION PROGRAM

Manager: Bruce Kimmel (Bldg 7078-A, MS 6402

$574-5969$

$615 / 576-0311$

Deputy Manager: Lacy Hyde (Bldg 7078-C, MS 6402)

$574-5969$

615/576-1579

Site Remediation Manager: Sid Garland

574-5969

$615 / 576-8581$

Clinch River Program Manager: Bob Cook

$576-8646$

$615 / 574-7319$

\section{WASTE MANAGEMENT}

ENVIRONMENTAL RESTORATION AND WASTE MANAGEMENT PROGRAMS

Vice President: J. Robert Merriman (Located at K-25 Site)

$574-9936$

$615 / 576-5800$

CENTRAL WASTE MANAGEMENT DIVISION

Director: Charles Frye (Bldg 1037, MS 7357)

$576-6222$

$615 / 574-9999$

ORNL WASTE MANAGEMENT AND REMEDIAL ACTIONS DIVISION

Director: Leonard McNeese (Bldg 3047, MS 6023)

$574-6226$

$615 / 574-7456$

WASTE MANAGEMENT PROGRAMS

Manager: Courtney Manrod (Bldg 3047, MS 6023)

$574-6226$

$615 / 574-7043$

DECONTAMINATION/DECOMMISSIONING PROGRAM

Manager: Thomas Burwinkle (Bldg 3001, MS 6028)

$576-7618$

$615 / 576-0224$

REMEDIAL ACTION SECTION

Head: H. Ray Gaddis (Bldg 3001, MS 6029)

$576-7618$

$615 / 576-0224$

WASTE MANAGEMENT OPERATIONS SECTION

Head: Thomas Scanlan (Bidg 3042, MS 6021)

$576-0105$

$615 / 574-4562$

ENVIRONMENTAL PROGRAMS COORDINATION SECTION

Head: Leroy Stratton (BIdg 3042, MS 6020)

574-0105

$615 / 576-0504$

ENVIRONMENTAL PROJECTS SECTION

Head: Robert Mason (BIdg 3047, MS 6023)

$576-4226$

$615 / 574-1365$

*DIAL AREA CODE

**RCRA/CERCLA/TSCA REGULATORY RESPONSE LEAD

$\mathrm{OR}-8$ 


\section{K-25 SITE OFFICE - DOE}

200 ADMINISTRATION ROAD

OAK RIDGE, TENNESSEE, 37831

P.O. BOX 2001

OAK RIDGE, TENNESSEE 37831-7136

FAX * COMMERCIAL

OFFICE OF ASSISTANT MANAGER FOR ENVIRONMENTAL

RESTORATION \& WASTE MANAGEMENT

Assistant Manager: William Adams

576-3770

$615 / 576-0742$

Deputy Assistant Manager: Bryan Walker

576-3770

615/576-0716

\section{ENVIRONMENT}

SITE OPERATIONS

Site Manager: Tom Tison

$574-4724 \quad 615 / 576-1974$

K-25 SITE - ENVIRONMENTAL (SITE OPERATIONS)

Richard Frounfelker ${ }^{\star \star}$

$574-4724 \quad 615 / 576-2990$

\section{ENVIRONMENTAL RESTORATION}

DEPUTY ASSISTANT MANAGER FOR ENVIRONMENTAL RESTORATION \& WASTE MANAGEMENT:

Deputy Assistant Manager: Bryan Walker

$576-3770$

615/576-0716

ENVIRONMENTAL RESTORATION DIVISION

Director: Robert Sleeman

$576-7042$

$615 / 576-3534$

OAK RIDGE REMEDIATION BRAINCH

Chief: W. Nelson Lingle

$576-7042 \quad 615 / 576-5580$

RESTORATION PROGRAM INTEGRATION BRANCH

Chief: Clayton Gist

$576-7042 \quad 615 / 576-6821$

*DIAL AREA CODE

**RCRA/CERCLA/T SCA REGULATORY RESPONSE LEAD

OR - 9 


\section{WASTE MANAGEMENT}

OFFICE OF ASSISTANT MANAGER FOR ENVIRONMENTAL

RESTORATION \& WASTE MANAGEMENT

Deputy Assistant Manager: Bryan Walker

WASTE MANAGEMENT DIVISION

Director: Larry Radcliffe

WASTE PROGRAM INTEGRATION BRANCH

Chief: Marianne Heiskell

WASTE OPERATIONS \& TECHNOLOGY DEVELOPMENT BRANCH

Chief: Douglas Underwood 
K-25 SITE - MMES

HIGHWAY 58

OAK RIDGE, TENNESSEE 37831

P.O. BOX 2003

OAK RIDGE, TENESSEE 37831

FAX*

576-5997

MANAGER: LINCOLN HALL

\section{ENVIRONMENT}

HEALTH AND SAFETY DIVISION

Director: Dave Milan

576-7489

$615 / 576-6833$

ENVIRONMENTAL MANAGEMENT DIVISION

Director: Larry Long

$576-7662$

$615 / 574-8222$

\section{ENVIRONMENTAL RESTORATION}

ENVIRONMENTAL RESTORATION PROGRAM

Manager: Joseph Haymore

$576-4048$

$615 / 574-9352$

K-25 SITE ASSESSMENTS

Manager: Robert Holmes

K-25 SITE REMEDIATION

Manager: Rolf Migun (Acting)

WASTE MANAGEMENT

WASTE MANAGEMENT DIVISION

Director: H. Dave Whitehead

DEPARTMENT OF WASTE MANAGEMENT ENGINEERING AND COMPL!ANCE

Department Head: Chuck Scheibly

QUALITY SERVICES DEPARTMENT

Department Head: Randy Mehlon
$576-6831$

$615 / 574-9700$

$576-4048$

$615 / 576-6473$

$576-4048$

$615 / 576-6088$

$576-6831$

$615 / 576-6831$

574-9399

$615 / 574-6830$ 
Y-12 SITE OFFICE - DOE

200 ADMINISTRATION ROAD

OAK RIDGE, TENNESSEE 37831

P.O. BOX 2001

OAK RIDGE, TENNESSEE 37831-8555

FAX*

COMMERCIAL

MANAGER: ROBERT SPENCE

574-9370

$615 / 576-9850$

\section{ENVIRONMENT}

ENVIRONMENT, SAFETY \& HEALTH BRANCH

Chief: Dan Hoag

576-9852

615/576-0511

Environmental Scientist: Brian DeMonia**

$576-8010 \quad 615 / 576-9254$

ENVIRONMENTAL RESTORATION

ENVIRONMENTAL RESTORATION DIVISION

Director: Robert Sleeman

576-7042

$615 / 576-0715$

ENVIRONMENTAL RESTORATION BRANCH

Clilef: Nelson Lingle

$576-7042$

$615 / 576-5580$

SITE REPRESENTATIVE

Sherry Lankford

$576-7042$

$615 / 576-9555$

\section{WASTE MANAGEMENT}

WASTE MANAGEMENT DIVISION

Director: Larry Radcliffe

576-5333

$615 / 576-9212$

WASTE OFERATIONS \& TECHNOLOGY DEVELOPMENT BRANCH

Chief: Doug Underwood

$576-5333$

$615 / 576-0728$

SITE REPRESENTATIVE

Joy Sager

$576-0850$

$615 / 576-0850$ 
Y-12 PLANT - MMES

BUILDING 9704-2, MS-8010

OAK RIDGE, TENNESSEE, 37831

P.O. BOX 2009

OAK RIDGE, TENNESSEE $37831-8010$

FAX* COMMERCIAL

MANAGER: D. JEFFERY BOSTOCK

576-7935

$615 / 574-2527$

DEFENSE PROGRAMS MANAGER: MARGARET MORROW

574-3742 615/574-2112

\section{ENVIRONMENT}

HEALTH, SAFETY \& ENVIRONMENTAL ACCOUNTABILITY

Director: Todd Butz

$574-9041 \quad 615 / 574-1052$

HEALTH AND ENVIRONMENT DEPARTMENT

Manager: Ron Keyser

$574-9041 \quad 615 / 574-1711$

ENVIRONMENTAL MANAGEMENT DEPARTMENT

Manager: Jimmy Stone

$574-9041 \quad 615 / 574-6911$

\section{ENVIRONMENTAL RESTORATION}

ENVIRONMENTAL RESTORATION AND WASTE MANAGEMENT PROGRAM

Vice President: J. Robert Merriman (Located at K-25 Site)

$574-9936 \quad 615 / 576-5800$

Deputy Manager: Finnis Patton (Bldg K1001, MS 7169)

$576-4014 \quad 615 / 574-2991$

Special Assistant: Lanny Bates (Bldg K1001, MS 7169)

576-4014 615/574-8995

ENVIRONMENTAL RESTORATION DIVISION

Director: Fran DeLozier (Bldg 9983-AG, MS 8247)

$574-9538 \quad 615 / 576-3712$

Y-12 ENVIRONMENTAL RESTORATION PROGRAM OFFICE

Manager: William Brown (Bldg 9983.AG, MS 8247)

Acting Deputy Director: Marc Norris

576-3808 615/576-5706

Site Restoration Manager: Bob Hill

$576-3808 \quad 615 / 576-5366$

Program Planning and Controls Manager: Jim Siberell

576-3808 615/574-2795

576-3808 $615 / 576-3978$

*DIAL AREA CODE

**RCRA/CERCLA/TSCA REGULATORY RESPONSE LEAD

OR -13 
WASTE MANAGEMENT

WASTE MANAGEMENT DIVISION

Director: Jim Heiskell (Bldg 9983-71, MS 8180)

$576-3946$

$615 / 574-8444$

WASTE TRANSPORTATION, STORAGE AND DISPOSAL DEPARTMENT

Head: Ken Dellus

$574-6855 \quad 615 / 576-4965$

WASTE MANAGEMENT ENGINEERING

Head: Iver Jeter

$576-3946 \quad 615 / 576-6453$

COMPLIANCE MANAGEMENT AND SYSTEMS ENGINEERING

Head: Dave McCune

$576-3946$

$615 / 576-5280$

WASTE TREATMENT OPERATIONS

Head: N. George McRae

576-3946 $\quad 615 / 574-6825$

*DIAL AREA CODE

**RCRA/CERCLA/TSCA REGULATORY RESPONSE LEAD

OR -14 
PADUCAH SITE OFFICE - DOE

5600 HOBBS ROAD

PADUCAH, KENTUCKY 42001

P.O. BOX 1410

PADUCAH, KENTUCKY 42002

FAX* COMMERCIAL

MANAGER: DONALD BOOHER

441-6803 502/441-6801

ENVIRONMENT

ENVIRONMENT SAFETY \& HEALTH BRANCH (PROPOSED)

Deputy Plant Manager: Jimmie Hodges

$441-6803 \quad 502 / 441-6806$

\section{ENVIRONMENTAL RESTORATION}

ENVIRONMENTAL RESTORATION PROGRAM

Manager: Robert Edwards

$441-6803 \quad 502 / 441-6817$

\section{WASTE MANAGEMENT}

ENVIRONMENT SAFETY \& HEALTH BRANCH

Deputy Plant Manager: Jimmie Hodges

$441-6803$

$502 / 441-6806$

WASTE MANAGEMENT

David Tidwell**

$441-6803 \quad 502 / 441-6807$

*DIAL AREA CODE

**RCRA/CERCLA/TSCA REGULATORY RESPONSE LEAD

OR -15 
PADUCAH GASEOUS DIFFUSION PLANT (PGDP) - MMES

5600 HOBB ROAD

PADUCAH, KENTUCKY 42001

P.O. BOX 1410

PADUCAH, KENTUCKY 42002

FAX*

441-6338

MANAGER: STEVE POLSTON

ENVIRONMENT

DEPUTY PLANT MANAGER (ESH): Howard Pulley

ENVIRONMENTAL AND WASTE MANAGEMENT DIVISION

Manager: Steve Shell

ENVIRONMENTAL COMPLIANCE

Deputy Manager: C. Gail Giltner

ENVIRONMENTAL RESTORATION

SITE MANAGER: Dennis Cope

\section{WASTE MANAGEMENT}

ENVIRONMENTAL AND WASTE MANAGEMENi DIVISION

Manager: Steve Shell

WASTE MANAGEMENT

Linda Beach
441-6049 502/441-6126

$441-6559 \quad 502 / 441-6466$

$441-6559 \quad 502 / 441-6319$

*DIAL AREA CODE

**RCRA/CERCLA/TSCA REGULATORY RESPONSE LEAD

OR - 16 
PORTSMOUTH SITE OFFICE - DOE

3930 U.S. RTE 23

PIKETON, OHIO 45661

P.O. BOX 900

PIKETON, OHIO 45661

FAX* COMMERCIAL

MANAGER: EUGENE GILLESPIE

897-2982 614/897-5010

ENVIRONMENT

Melda Rafferty

897-3572 614/897-3821

ENVIRONMENTAL RESTORATION

SITE REPRESENTATIVE: David Dollins

897-3572 614/897-3860

WASTE MANAGEMENT

Melda Rafferty**

897-2982

$614 / 897-3821$

*DIAL AREA CODE

**RCRA/CERCLA/TSCA REGULATORY RESPONSE LEAD

OR -17 
PORTSMOUTH GASEOUS DIFFUSION PLANT (PORTS) - MMES

3930 U.S. ROUTE 23

PIKETON, OHIO 45661

P.O. BOX 628

PIKETON, OHIO 45661

FAX

897-2909

PLANT MANAGER: RALPH DONNELLY

\section{ENVIRONMENT}

ENVIRONMENT \& SUPPORT SERVICES

Manager: David Taylor

ENVIRONMENT, SAFETY \& HEALTH

Manager: Clarence Sheward

ENVIROMENTAL CONTROL

Department Head: Richard Blake

ENVIRONMENTAL RESTORATION

ENVIRONMENT \& SUPPORT SERVICES

Manager: David Taylor

ENVIRONMENT, SAFETY \& HEALTH

Manager: Clarence Sheward

REMEDIAL ACTION

Department Head: Richard Snyder

\section{WASTE MANAGEMENT}

DEPUTY PLANT MANAGER:

John Shoemaker

WASTE MANAGEMENT

Department Head: Jeffrey Hedges
897-2909

$614 / 897-2700$

$897-2221$

$614 / 897-2268$

897-3752

$614 / 897-2145$

897-2909

$614 / 897-2700$

$897-2221$

$614 / 897-2268$

897-3752

$614 / 897-2322$

897-3720

$617 / 897-2268$

897-3846

$617 / 897-4052$

* DIAL aREA code

**RCRA/CERCLA/TSCA REGULATORY RESPONSE LEAD 
FORMERLY UTILIZED SITES REMEDIAL ACTION PROGRAM (FUSRAP) - DOE 105 LEA WAY

BUILDING 2714-H

OAK RIDGE, TENNESSEE, 37831

P.O. BOX 2001

OAK RIDGE, TENNESSEE $37831-8723$

FAX * COMMERCIAL

PROJECT MANAGER: LESTER PRICE

576-0956

615/576-0948

DEPUTY PROJECT MANAGER: WILLIAM SEAY

576-0956 615/576-1830

Site Manager, Missouri/Ohio Sites: David Adler

576-0956 615/576-9634

Site Manager, New Jersey Sites: Susan Cainge

576-0956

$615 / 576-5724$

Site Manager, New York Sites: Ronald Kirk

576-0956

615/576-7477

Site Manager, Other Sites: Teresa Perry

576-0956

$615 / 576-8956$

Site Manager, Project Support Group: James Hart

576-0956

615/576-0707 
BECHTEL NATIONAL, INC.

151 LAFAYETTE DRIVE

OAK RIDGE, TENNESSEE, 37830

P.O. BOX 350

OAK RIDGE, TENNESSEE $37831-0350$

FAX*

COMMERC.IAL

VICE PRESIDENT AND FUSRAP PROGRAM MANAGER:

GALE HOVEY

\section{ENVIRONMENT}

DEPUTY PROGRAM MANAGERS:

Planning: Richard Harbert Operations: G. Phil Crotwell

ENVIRONMENTAL, HEALTH, SAFETY \& WASTE MANAGEMENT

Manager: Thomas Morris

\section{ENVIRONMENTAL RESTORATION}

NEW YORK FUSRAP SITES

Project Manager: Cathy Hickey

NEW JERSEY FUSRAP SITES

Project Manager: Steven Liedle

MISSOURI AND OHIO SITES

Project Manager: Gerald Palau

REGULATORY COMPLIANCE

Project Manager: Edward McNamee
$576-4898$

$576-4898$

615/576-3998

$615 / 576-9467$

$576-4898$

615/576-3355

$576-4898 \quad 615 / 576-1677$

$576-4898 \quad 615 / 576-3997$

$576-4898$

$615 / 576-3859$

*DIAL AREA CODE

**RCRA/CERCLA/TSCA REGULATORY RESPONSE LEAD

OR -20 
WELDON SPRING SITE OFFICE - DOE

7295 HIGHWAY 94 SOUTH

ST. CHARLES, MISSOURI 63304

FAX * COMMERCIAL

MANAGER: STEVE MCCRACKEN

477-0739

314/441-8978

ENVIRONMENT

Environmental Engineer: Alan Gibson**

477-0739 $\quad 314 / 441-8978$

ENVIRONMENTAL RESTORATION

Project Manager: Steve McCracken**

477-0739 $\quad 314 / 441-8978$

WASTE MANAGEMENT

Environmental Engineer: Alan Gibson

477-0739 $\quad 314 / 441-8978$ 
WELDON SPRING SITE - MK-FERGUSON COMPANY

7295 HIGHWAY 94 SOUTH

ST. CHARLES, MISSOURI 63304

FAX*

DIRECTOR: JAMES POWERS

\section{ENVIRONMENT}

ENVIRONMENTAL, SAFETY \& HEALTH DEPARTMENT

Manager: Steve Green

PERSONNEL PROTECTION

Manager: Dan Hoffman

ENVIRONMENTAL PROTECTION

Manager: Jim Meier

ENVIRONMENTAL RESTORATION

Project Director: James Powers

WASTE MANAGEMENT

COMPLIANCE DEPARTMENT

Manager: Dave Hixson

WASTE MANAGEMENT SECTION

Manager: Lannis Philips
447-0803

314/441-8086

477-0803

$314 / 441-8086$

477-0803

$314 / 441-8086$

477-0803

$314 / 441-8086$

477-0803

$314 / 441-8086$

477-0803

$314 / 441-8086$ 
Pittsburgh Naval Reactors (PNR) 
PITTSBURGH NAVAL REACTORS OFFICE - DOE

814 PITTSBURGH-MCKEESPORT BOULEVARD

WEST MIFFLIN, PENNSYLVANIA 15122

P.O. BOX 109

WEST MIFFLIN, PENNSYLVANIA 15122-0109

FAX * COMMERCIAL

GENERAL MANAGER: CARL GADDIS

476-7310 $412 / 476-7200$

\section{ENVIRONMENT}

OPERATIONS

Assistant Manager: David Curtis

$476-7310 \quad 412 / 476-7220$

ENVIRONMENT, SAFETY \& HEALTH

Project Officer: Eart Shollenberger ${ }^{\star \star}$

476-7310

412/476-7290

ENVIRONMENTAL RESTORATION

OPERATIONS

Assistant Manager: David Curtis

$476-7310 \quad 412 / 476-7220$

ENVIRONMENT, SAFETY \& HEALTH

Project Officer: Earl Shollenberger

$476-7310$

412/476-7290

WASTE MANAGEMENT

OPERATIONS

Assistant Manager: David Curtis

476-7310

412/476-7220

ENVIRONMENT, SAFETY \& HEALTH

Project Officer: Earl Shollenberger

476-7310

412/476-7290

* dial area code

* RCRA/CERCLA/TSCA REgULATORY RESPONSE LEAD

PNR - 1 
WESTINGHOUSE ELECTRIC CORPORATION

BETTIS ATOMIC POWER LABORATORY

814 PITTSBURGH-MCKEESPORT BOULEVARD

WEST MIFFLIN, PENSYLVANIA 15122

P.O. BOX 79

WEST MIFFLIN, PENNSYLVANIA 15122-0079

FAX* COMMERCIAL

GENERAL MANAGER: BYRON RUTH

476-5151 412/476-5111

ENVIRONMENT

RADIOLOGICAL CONTROLS AND ENGINEERING

Manager: Nancy Harris**

476-5151

$412 / 476-6550$

ENVIRONMENTAL RESTORATION

RADIOLOGICAL CONTROLS AND ENGINEERING

Manager: Nancy Harris

$476-5151 \quad 412 / 476-6550$

WASTE MANAGEMENT

RADIOLOGICAL CONTROLS AND ENGINEERING

Manager: Nancy Harris

476-5151 412/476-6550

*DIAL AREA CODE

*\#RCRA/CERCLA/TSCA REGULATORY RESPONSE LEAD

PNR - 2 
Power Administrations (PA) 
ALASKA POWER ADMINISTRATION

2770 SHERWOOD LANE, SUITE 2B

JUNEAU, ALASKA 99802

FAX* COMMERCIAL

ADMINISTRATOR: MICHAEL DEIHL

586-7270 907/586-7405

ENVIRONMENTAL

ENVIRONMENTAL

Branch Chief: Rob Waldman ${ }^{\star \star}$

586-7270 907/586-7405

ES\&H Specialist: Teresa Eade

586-7270 907/586-7405

\section{ENVIRONMENTAL RESTORATION}

ENVIRONMENTAL

Branch Chief: Rob Waldman

586-7270 907/586-7405

ES\&H Specialist: Teresa Eade

586-7270 907/586-7405

WASTE MANAGEMENT

ENVIRONMENTAL

Branch Chief: Rob Waldman

586-7270

907/586-7405

ES\&H Specialist: Teresa Eade

586-7270

907/586-7405

*DIAL AREA CODE

**RCRA/CERCLA/TSCA REGULATORY RESPONSE LEAD

PA - 1 
BONNEVILLE POWER ADMINISTRATION

905 N.E. 11TH AVENUE

PORTLAND, OREGON 97232

P.O. BOX 3621

PORTLAND, OREGON 97208-3621

\section{FAX* COMMERCIAL}

ADMINISTRATOR: RANDALL HARDY

230-5211 503/230-5101

ENVIRONMENT

ENVIRONMENT

Assistant Administrator: Alexandra Smith

230-5211 503/230-5137

\section{ENVIRONMENTAL RESTORATION}

ENVIRONMENT

Assistant Administrator: Alexandra Smith

230-5211 503/230-5137

THIRD PARTY SITES

Stephen Sander **

230-5211 503/230-4724

OFFICE OF OPERATIONS, MAINTENENCE \& CONSTRUCTION

Assistant Administrator: James Jones

230-4718 503/230-3101

DIVISION OF SYSTEM MAINTENANCE

Director: Frederick Johnson

$690-2958 \quad 206 / 690-2001$

ENVIRONMENTAL PROTECTION BRANCH

Robert Kiser

690-2562 206/690-2044

*DIAL AREA CODE

**RCRA/CERCLA/TSCA REGULATORY RESPONSE LEAD

PA - 2 


\section{WASTE MANAGEMENT}

\section{ENVIRONMENT}

Assistant Administrator: Alexandra Smith

RCRA (OVERALL)

Shannon Allison

$230-5211 \quad 503 / 230-3599$

OFFICE OF MANAGEMENT SERVICES

Assistant Administrator: Steve Ailshie

$230-5211 \quad 503 / 230-4965$

DIVISION OF MATERIALS \& PROCUREMENT

Director: Peter Stemple (Acting)

$230-4550 \quad 503 / 230-4163$

UTILIZATION \& DISPOSAL BRANCH

Chief: Vern Shipe

690-2432 206/690-5769

*DIAL AREA CODE

**RCRA/CERCLA/TSCA REGULATORY RESPONSE LEAD

PA - 3 
SOUTHEASTERN POWER ADMINISTRATION

SAMUEL ELBERT BUILDING

ELBERTON, GEORGIA 30635

FAX*: COM 706/283-9928

FAX*

ADMINISTRATOR: JOHNNY MCALLISTER

ENVIRONMENT

POWER RESOURCES DIVISION

Director: Jim Lloyd**

HYDRAULIC ENGINEER

Tony Truett

ENVIRONMENTAL RESTORATION

POWER RESOURCES DIVISION

Director: Jim Lloyd

HYDRAULIC ENGINEER

Tony Truett

\section{WASTE MANAGEMENT}

POWER RESOURCES DIVISION

Director: Jim Lloyd

HYDRAULIC ENGINEER

Tony Truett
283-9928

706/283-9911

$238-9928$

706/283-9911

$238-9928$

$706 / 283-9911$

238-9928

$706 / 283-9911$

238-9928

706/283-9911

238-9928

$706 / 283-9911$

*DIAL AREA CODE

*\#RCRA/CERCLA/TSCA REGULATORY RESPONSE LEAD

$$
\text { PA - } 4
$$


SOUTHWESTERN POWER ADMINISTRATION

WILLIAMS CENTRE TOWER 1

1 W. 3RD STREET

TULSA, OKLAHOMA 74103

P.O. BOX 1619

TULSA, OKLAHOMA 74101

FAX* COMMERCIAL

ADMINISTRATOR: JOHN SHAFER

$581-7530 \quad 918 / 581-7476$

\section{ENVIRONMENT}

OFFICE OF ENVIRONMENTAL SAFETY, HEALTH \& SECURITY

Manager: Leo Mucherheide

$581-7530 \quad 918 / 581-7528$

\section{ENVIRONMENTAL RESTORATION}

OFFICE OF ENVIRONMENTAL SAFETY, HEALTH \& SECURITY

Manager: Leo Mucherheide

$581-7530$

$918 / 581-7528$

WASTE MANAGEMENT

OFFICE OF ENVIRONMENTAL SAFETY, HEALTH \& SECURITY

Manager: Leo Mucherheide

$581-7530$

$918 / 581-7528$

Environmental Protection Specialist: David Dossett**

$581-7530$

$918 / 581-6819$ 
WESTERN AREA POWER ADMINISTRATION

1627 COLE BUILDING

BUILDING 18

GOLDEN, COLORADO 80401

P.O. BOX 3402

GOLDEN, COLORADO 80401-0092

FAX* COMMERCIAL

ADMINISTRATOR: WILLIAM CLAGETT

231-1632 303/231-1513

ENVIRONMENT

CONSERVATION, ENVIRONMENT \& SAFETY

Assistant Administrator: Vicki Ponce (Acting)

231-1632 $303 / 231-7142$

DIVISION OF ENVIRONMENTAL AFFAIRS

Director: William Karsell

$231-7967 \quad 303 / 231-1706$

\section{ENVIRONMENTAL RESTORATION}

CONSERVATION, ENVIRONMENT \& SAFETY

Assistant Administrator: Vicki Ponce (Acting)

$231-1632 \quad 303 / 231-7142$

DIVISION OF ENVIRONMENTAL AFFAIRS

Director: William Karsell

231-7967

$303 / 231-1706$

ENVIRONMENTAL COMPLIANCE

Manager: Ken Mathias**

$231-7967$

$303 / 231-7401$

WASTE MANAGEMENT

CONSERVATION, ENVIRONMENT \& SAFETY

Assistant Administrator: Vicki Ponce (Acting)

231-1632

$303 / 231-7142$

DIVISION OF ENVIRONMENTAL AFFAIRS

Director: William Karsell

$231-7967 \quad 303 / 231-1706$

ENVIRONMENTAL COMPLIANCE

Manager: Ken Mathias

$231-1632$

$303 / 231-7401$

*DIAL AREA CODE

**RCRA/CERCLA/TSCA REGULATORY RESPONSE LEAD

PA - 6 
Richland Field Office (RL) 
RICHLAND FIELD OFFICE - DOE

825 JADWIN AVENUE

RICHLAND, WASHINGTON 99352

P.O. BOX 550

RICHLAND, WASHINGTON 99352

FAX * COMMERCIAL

MANAGER: JOHN WAGONER

376-4789 509/376-7395

\section{ENVIRONMENT}

OFFICE OF DEPUTY MANAGER FOR SITE RESOURCES

Deputy Manager: J. Phil Hamric

$376-4789 \quad 509 / 376-7397$

OFFICE OF PERFORMANCE ASSESSMENT

Director: R. Pierre Saget

$376-6621 \quad 509 / 376-2611$

SAFETY, ENVIRONMENT \& SECURITY ASSESSMENT BRANCH

Chief: Gerry Bell

$376-6621 \quad 509 / 376-0680$

OFFICE OF ENVIRONMENTAL ASSURANCE PERMITS \& POLICY

Program Manager: Ronald Izatt

$376-7818 \quad 509 / 376-5441$

TRI-PARTY AGREEMENT BRANCH

Chief: Steven Wisness

$376-7818 \quad 509 / 376-1906$

REGULATORY POLICY, PLANNING \& ANALYSIS BRANCH

Chief: Robert Holt**

$376-7818 \quad 509 / 376-1906$

REGULATORY PERMITS BRANCH

Chief: Jim Rasmussen

376-7818 509-376-1906

*DIAL AREA CODE

**RCRA/CERCLA/TSCA REGULATORY RESPONSE LEAD

RL -1 
ENVIRONMENTAL RESTORATION

OFFICE OF THE DEPUTY MANAGER FOR ENVIRONMENTAL RESTORATION AND PROJECTS

Deputy Manager: Willis Bixby/John Wagoner (Acting)

$376-4789 \quad 509 / 376-0024$

OFFICE OF ASSISTANT MANAGER FOR ENVIRONMENTAL MANAGEMENT

Assistant Manager: Leo Little

$376-4360$

$509 / 376-6628$

ENVIRONMENTAL RESTORATION DIVISION

Director: Elizabeth Bracken

$376-4360$

$509 / 376-7277$

ENVIRONMENTAL PROGRAMS BRANCH

Chief: Roger Freeberg

$376-4360 \quad 509 / 376-7167$

ENVIRONMENTAL ASSESSMENT BRANCH

Chief: Susan Clark

$376-7818 \quad 509 / 376-9333$

ENVIRONMENTAL REMEDIATION BRANCH

Chief: Julie Erickson* *

$376-7818 \quad 509 / 376-3603$

\section{WASTE MANACEMENT}

OFFICE OF ASSISTANT MANAGER FOR OPERATIONS

Assistant Manager: John Hunter

376-0695 509/376-7434

WASTE MANAGEMENT DIVISION

Director: Ken Bracken/June Hennig (Acting)

376-0695

$509 / 376-1366$

WASTE OPERATIONS BRANCH

Chief: Jim Daily/Rudy Guercia (Acting) ${ }^{\star *}$

376-1926

$509 / 376-7721$

$509 / 376-5494$

-DIAL AREA CODE

**RCRACERCLATSCA REGULATORY RESPONSE LEAD

$\mathrm{RL} \cdot \mathbf{2}$ 
BATTELLE MEMORIAL INSTITUTE

PACIFIC NORTHWEST LABORATORIES (PNL)

902 BATTELLE BOULEVARD

RICHLAND, WASHINGTON 99352

P. O. BOX 999

RICHLAND, WASHINGTON 99352

375-6844

DIRECTOR: WILLIAM WILEY

ENVIRONMENT

FACILITIES AND OPERATIONS

Director: Tom Chikalla

LABORATORY SAFETY

Manager: Glenn Hoenes

ENVIRONMENTAL COMPLIANCE SECTION

Manager: Harold Tilden

\section{ENVIRONMENTAL RESTORATION}

ENVIRONMENTAL RESTORATION \& WASTE MANAGEMENT

Director: Joe Spencer

OFFICE OF HANFORD ENVIRONMENT

Manager: Bob Gray

ENVIRONMENTAL MANAGEMENT OPERATIONS

Manager: James Doesburg

OFFICE OF ENVIRONMENTAL TECHNOLOGY

Manager: Mark Hanson
FAX*

376-7885

509/376-0499

$375-4343$

$509 / 375-4331$

COMMERCIAL

$509 / 375-2201$

$509 / 376-2239$

$509 / 376-1187$

$375-6978 \quad 509 / 375-2937$

$376-9037 \quad 509 / 376-0341$

376-3606 $\quad 509 / 375-6812$

*DIAL AREA CODE

**RCRA/CERCLA/TSCA REGULATORY RESPONSE LEAD

RL - 3 


\section{WASTE MANAGEMENT}

FACILITIES AND OPERATIONS

Director: Tom Chikalla

372-0128

509/376-2239

LABORATORY SAFETY

Manager: Glenn Hoenes

$376-6663$

509/376-1187

WASTE MANAGEMENT SECTION

Manager: Bill Bjorkland

376-7885

$509 / 376-4781$

*DIAL AREA CODE

**RCRA/CERCLA/TSCA REGULATORY RESPONSE LEAD

$\mathrm{RL}-4$ 
WESTINGHOUSE HANFORD CO.

1100 JADWIN STREET

RICHLAND, WASHINGTON 99352

P.O. BOX 1970

RICHLAND, WASHINGTON 99352

FAX * COMMERCIAL

PRESIDENT: TOM ANDERSON (MAIL STOP 8301)

376-3440 509/376-5107

ENVIRONMENT

ENVIRONMENTAL, DIVISION

Director: Richard Wojtesck

376-5370 509/376-700

ENVIRONMENTAL SAFETY

Manager: David Watson

373-3324

$509 / 373-3250$

ENVIRONMENTAL ENGINEERING STUDIES

Manager: John Dorian

$376-2816$

$509 / 376-5597$

ENVIRONMFNTAL COMPLIANCE ASSURANCE

Manager: Joseph Rivera

$376-9110$

$509 / 376-1449$

ENVIRONMENTAL COMPLIANCE SUPPORT - 100 AREA

Manager: Ken Gano

373-3046 509/373-4949

EFFLUENT EMIISSION MONITORING - 200 AREA

Manager: Larry Diediker

373-5394 509/373-1716

RCRA COMPLIANCE SUPPORT

Manager: Eric Greager

376-2816 509/376-3132

*DIAL AREA CODE

**RCRA/CERCLA/TSCA REGULATORY RESPONSE LEAi

RL - 5 


\section{ENVIRONMENTAL RESTORATION}

EXECUTIVE VICE PRESIDENT: RALPH DISIBIO

$509 / 376-7803$

RESTORATION \& REMEDIATION DEPARTMENT

Director: Hank McGuire

$376-0119 \quad 509 / 376-1400$

ENVIRONMENTAL DIVISION

Manager: Richard Wojtasek

$376-5370$

$509 / 376-7000$

REGULATORY SUPPORT

George Jackson

376-2816

$509 / 376-9315$

ENVIRONMENTAL ENGINEERING

Men Lauterbach

$376-6476$

$509 / 376-5257$

DECOMMISSIONING ENGINEERING

Michael Mihalic

$376-5370$

509/373-1382

ENVIRONMENTAL RESTORATION OPERATIONS

Michael Hughes

$373-2280 \quad 509 / 373-1184$

WASTE MANAGEMENT

RESTORATION \& REMEDIATION DEPARTMENT

Director: Hank McGuire

$376-0119 \quad 509 / 376-1400$

SOLID WASTE CISPOSAL

Manager: William Hamilton

$376-5425 \quad 509 / 376-4596$

$R L-6$ 
Rocky Flats (RF) 
ROCKY FLATS OFFICE: - DOE

ROCKY FLATS PLANT

ROCKY FLATS, COLORADO 80401

\section{P.O. BOX 928}

GOLDEN, COLORADO 80401

FAX * COMMERCIAL

MANAGER: ROBERT NELSON, JR.

966-6054

303/966-2025

\section{ENVIRONMENT}

ENVIRONMENTAL MANAGEMENT

Assistant Manager: James Hartman

966-2995

$303 / 966-5918$

WASTE MANAGEMENT \& ENVIRONMENT DIVISION

Director: Thomas Lukow

966-2256

$303 / 966-4561$

ENVIRONMENTAL MONITORING BRANCH

Mark Van Der Puy

966-2256 303/966-2473

\section{ENVIRONMENTAL RESTORATION}

ENVIRONMENTAL MANAGEMENT

Assistant Manager: James Hartman

966-2995 303/966-5918

ENVIRONMENTAL RESTORATION DIVISION

Director: Frazer Lockhart ${ }^{\star \star}$

$966-4871 \quad 303 / 966-7846$

*DIAL ARea code

**CERCLA REGULATORY RESPONSE LEAD

***RCRA/TSCA REGULATORY RESPONSE LEAD

RF - 1 
WASTE MANAGEMENT

ENVIRONMENTAL MANAGEMENT

Assistant Manager: James Hartman

966-2995 303/966-5918

WASTE MANAGEMENT AND ENVIRONMENT DIVISION

Director: Thomas Lukow

966-2256 303/966-4561

WASTE OPERATIONS BRANCH

Chief: Joe Wienand ${ }^{\star \star \star}$

966-2256 303/966-5926

WASTE PROGRAMS

John Rampe

966-2256 303/966-6246

*DIAL AREA CODE

**CERCLA REGULATORY RESPONSE LEAD

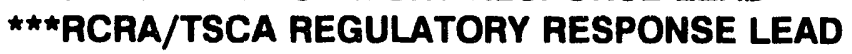

RF - 2 
EG \& G ROCKY FLATS

U.S. DOE

ROCKY FLATS PLANT

ROCKY FLATS, COLORADO 80401

P.O. BOX 464

GOLDEN, COLORADO 80402-0464

FAX: COMMERCIAL

GENERAL MANAGER: JAMES ZANE

966-4572 303/966-4361

\section{ENVIRONMENT}

ENVIRONMENTAL AND WASTE MANAGEMENT

Associate General Manager: Tim Hedahl

966-7' $183 \quad 303 / 966-4111$

ENVIRONMENTAL PROTECTION MANAGEMENT

Director: George Setlock

$966-8663$

$303 / 966-8576$

TECHNOLOGY DEVELOPMENT

Director: Tom Rising

$966-3407$

$303 / 966-4725$

\section{ENVIRONMENTAL RESTORATION}

ENVIRONMENTAL RESTORATION MANAGEMENT

Associate General Manager: Robert Benedetti

966-8538 303/966-8569

ENVIRONMENTAL SCIENCE AND ENGINEERING

Director: Tom Greengard (Acting)

$966-8556 \quad 303 / 966-8510$

REMEDIATION PROGRAMS

Director: Mike Arndt

966-8556 303/966-8509 


\section{WASTE MANAGEMENT}

ENVIRONMENTAL \& WASTE MANAGEMENT

Associate General Manager: Tim Hedahl

966-7183

$303 / 966-4111$

WASTE OPERATIONS

Director: Robert Morgan

966-6172 303/966-6012

WASTE PROGRAMS

Director: Allen Schubert

966-6406

$303 / 966-6595$

ANALYTICAL LABS

Jerry Zarret

966-4661

$303 / 966-5680$

*DIAL AREA CODE

* RCRA/CEFCLA/TSCA REGULATORY RESPONSE LEAD

RF - 4 
San Francisco Field Office (SF) 


\section{SAN FRANCISCO FIELD OFFICE - DOE OAKLAND FEDERAL BUILDINO 1301 CLAY STREET \\ OAKLAND, CALIFORNIA $94612-8200$}

FAX* COMMERCIAL

$637-2012 \quad 510 / 637-1800$

$637-2001 \quad 510 / 637-1587$

$637-2001 \quad 510 / 637-1592$

$637-2001 \quad 510 / 637-1594$

$637-2001 \quad 510 / 637-1634$

$637-2001 \quad 510 / 637-1619$

Director: Alex Dong (Livermore)

ENVIRONMENTAL RESTORATION BRANCH

Chief: Joseph Cullen**

$637-2001$

$510 / 637-1625$ 
WASTE MANACEMENT

ENVIRONMENTAL MANAGEMENT \& SUPPORT

Assistant Manager: James A. Davis, III.

$637-2001$

$510 / 637-1634$

ENVIRONMENTAL RESTORATION \& WASTE MANAGEMENT DIVISION

Director: Alex Dong (Livermore)

$637-2001$

510/637-1619

WASTE MANAGEMENT OPERATIONS BRANCH

Chief: Ray Corey (Livermore)

$637-2001$

$510 / 637-1588$ 
LAWRENCE BERKELEY LABORATORY (LBL)

1 CYCLOTRON ROAD

BERKELEY, CALIFORNIA 94720

FAX* COMMERCIAL

Director: CHARLES SHANK

486-6720 510/486-5111

\section{ENVIRONMENT}

ENVIRONMENTAL HEALTH \& SAFETY DIVISION

Division Director: David McGraw

486-7488

$510 / 486-5551$

Department Head: Kam Tung

486-7488

510/486-4048

ENVIRONMENTAL PROTECTION (PERMITS)

Group Leader: Ronald Pauer

486-4776

510/486-7614

$510 / 486-5251$

\section{ENVIRONMENTAL RESTORATION}

ENVIRONMENTAL HEALTH \& SAFETY DIVISION

Division Director: David McGraw

486-7488 510/486-5551

Department Head: Kam Tung

486-7488 510/486-4048

Group Leader: Iraj Javandel

486-5686 $\quad 510 / 486-4048$

\section{WASTE MANAGEMENT}

ENVIRONMENTAL HEALTH \& SAFETY DIVISION

Division Director: David McGraw

486-7488 510/486-5551

Department Head: Kam Tung

486-7488 510/486-4048

Group Leader: Tim Wan

486-4776 $\quad 510 / 486-7073$

510/486-5251

*DIAL AREA CODE

**RCRA/CERCLA/TSCA REGULATORY RESPONSE LEAD

$$
\text { SF - } 3
$$




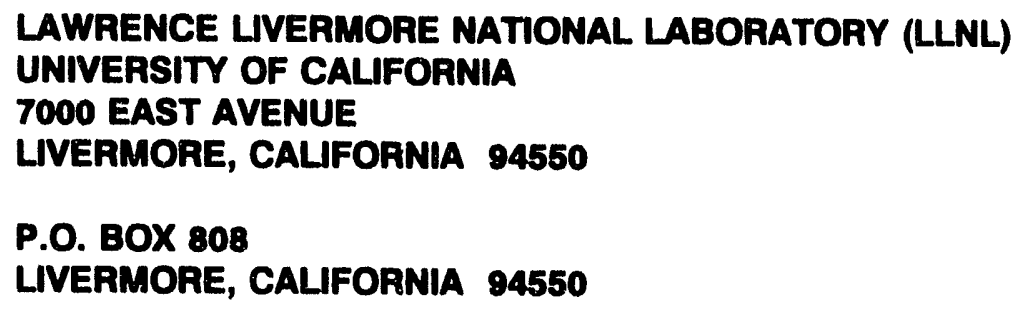

P.O. BOX 808

LIVERMORE, CALIFORNIA 94550

FAX*

$422-9667$

COMMERCIAL

DIRECTOR: JOHN NUCKOLLS (MAIL STOP L-1)

\section{ENVIRONMENT}

ENVIRONMENTAL PROTECTION DEPARTMENT

Department Head: Harry Galles

423-1395

$510 / 423-7983$

OPERATIONS \& REGULATORY AFFAIRS DIVISION

Division Leader: C. Susi Jackson

ENVIRONMENTAL MONITORING \& SURVEILLANCE DIVISION

Division Leader: William Isherwood

ENVIRONMENTAL RESTORATION

ENVIRONMENTAL PROTECTION DEPARTMENT

Department Head: Harry Galles

ENVIRONMENTAL RESTORATION DIVISION

Division Leader: William McConachie

\section{WASTE MANAGEMENT}

ENVIRONMENTAL PROTECTION DEPARTMENT

Department Head: Harry Galles

HAZARDOUS WASTE MANAGEMENT DIVISION

Division Leader: Dick Crawford
423-1395

$510 / 423-7983$

$422-3000$

$510 / 423-5058$

423-5754

510/423-3501

423-1395

$510 / 423-6745$

422-4195

$510 / 422-6309$

*DIAL AREA CODE

**RCRA/CERCLA/TSCA REGULATORY RESPONSE LEAD 
STANFORD LINEAR ACCELERATOR CENTER (SLAC)

2575 SAND HILL ROAD

MENLO PARK, CALIFORNIA 94025

P.O. BOX 4349

STANFORD, CALIFORNIA 94309

FAX*

COMMERCIAL

DIRECTOR: BURTON RICHTER

926-4500

415/926-2601

\section{ENVIRONMENT}

ENVIRONMENT, SAFETY \& HEALTH

Assoclate Director: Matthew Allen

926-3030

415/926-2820

ENVIRONMENT, SAFETY \& HEALTH

Assistant Director: Gary Warren

926-3030 415/926-3614

ENVIRONMENTAL PROTECTION \& WASTE MANAGEMENT

Department Head: Karen Holtemann

926-3175 415/926-4294

ENVIRONMENTAL RESTORATION

ENVIRONMENT, SAFETY \& HEALTH

Associate Director: Matthew Allen

$926-3030 \quad 415 / 926-2820$

ENVIRONMENT, SAFETY \& HEALTH

Assistant Director: Gary Warren

926-3030 $\quad 415 / 926-3614$

ENVIRONMENTAL PROTECTION \& WASTE MANAGEMENT

Department Head: Karen Holtemann

926-3175

$415 / 926-4294$

$$
\text { SF }-5
$$


WASTE MANAGEMENT

ENVIRONMENT, SAFETY \& HEALTH

Associate Director: Matthew Allen

926-3030

$415 / 926-4294$

ENVIRONMENT, SAFETY \& HEALTH

Assistant Director: Gary Warren

926-3030

$415 / 926-3614$

ENVIRONMENTAL PROTECTION \& WASTE MANAGEMENT

Department Head: Karen Holtemann

926-3175

$415 / 926-4294$

*DIAL AREA CODE

* RCRA/CERCLA/TSCA REGULATORY RESPONSE LEAD

SF -6 
ENERGY TECHNOLOGY ENGINEERING CENTER - DOE

U.S. DEPARTMENT OF ENERGY

SAN FRANCISCO FIELD OFFICE

1333 BROADWAY

OAKLAND, CALIFORNIA 94612

FAX* COMMERCIAL

ENVIRONMENT

ENVIRONMENTAL RESTORATION \& WASTE MANAGEMENT

DIVISION

ETEC Program Manager: Roger Liddle*ネ

273-6254

$510 / 273-7963$

ENVIRONMENTAL RESTORATION

ENVIRONMENTAL RESTORATION \& WASTE MANAGEMENT DIVISION

ETEC Program Manager: Roger Liddle

$273-6254 \quad 510 / 273-7963$

\section{WASTE MANAGEMENT}

ENVIRONMENTAL RESTORATION \& WASTE MANAGEMENT DIVISION

F.TEC Program Manager: Hannibal Joma

$422-0332$

$510 / 422-0883$

-DIAL AREA CCIDE

**RCRA/CERCLA,TSCA REgULATORY RESPONSE LEAD

SF $\cdot 7$ 
ENERGY TECHNOLOGY ENGINEEAING CENTER

ROCKWELL INTERNATIONAL - ROCKETDYNE DIVISION

6833 CANOGA AVENUE

CANOGA PARK, CALIFORNIA 91309

P.O. BOX 7930

CANOGA PARK, CALIFORNIA 91309

FAX*

COMMERCIAL

GENERAL MANAGER: D. CLARKE GIBBS

$586-5118$

818/586-5326

ENVIRONLIENT

ETEC PROGRAM MANAGER: Gerald Gaylord

$586-5118$

$818 / 586-5283$

HEALTH \& SAFETY

Director: Curtis Rozas

$710-5108$

$818 / 710-2275$

ENVIRONMENTAL RESTORATION

ETEC PFOGRAM MANAGER: Gerald Gaylord

$586-5118 \quad 818 / 586-5283$

HEALTH \& SAFETY

Director: Curtis Rozas

$710-5108 \quad 818 / 710-2275$

WASTE MANAGEMENT

LTEC PROGRAM MANAGER: Gerald Gaylord

$586-5118$

$818 / 586-5283$

HEALTH \& SAFETY

Director: Curtis Rozas

$710-5108 \quad 818 / 710-2275$

- DIAl aRea code

*\#RCRA/CERCLA/TSCA REGULATORY RESPONSE LEAD

$$
\text { SF }-8
$$


¿vannah River Field Office (SR) 
SAVANNAH RIVER FIELD OFFICE - DOE

SRS ROAD 1

BUILDING 746A

AIKEN, SOUTH CAROLINA 29808

P.O. BOX A

AIKEN, SOUTH CAROLINA 29802

FAX

725-1910

$725-1910$

$803 / 725-8074$

ENVIRONMENTAL DIVISION

Director: Steve Wright

ENVIRONMENTAL COMPLIANCE BRANCH

Chief: Ronald Peterson ${ }^{\star *}$

ENVIRONMENTAL PROGRAMS BRANCH

Chief: James Arnett

$725-8434$

$803 / 725-1645$

\section{ENVIRONMENTAL RESTORATION}

ENVIRONMENTAL RESTORATION \& WASTE MANAGEMENT

Assistant Manager: Leonard Sjostrom

725-3616

$803 / 725-1378$

ENVIRONMENTAL RESTORATION DIVISION

Director: Lewis Goidell

725-3616

803/725-3966

ENVIRONMENTAL RESTORATION BRANCH

Chief: Linda McClain

ENVIRONMENTAL COMPLIANCE AND PROGRAM MANAGEMENT BRANCH

Chief: Thomas Treger
$725-3616 \quad 803 / 725-8161$

725-3616
$803 / 725-1958$

*DIAL AREA CODE

**RCRA/CERCLA/TSCA REGULATORY RESPONSE LEAD 
ENVIRONMENTAL RESTORATION AND WASTE MANAGEMENT

Assistant Manager: Leonard Sjostrom

725-3616

$803 / 725-1378$

WASTE OPERATIONS AND TECHNICAL DIVISION

Director: Michael O'Rear

725-3616 803/725-5541

WASTE OPERATIONS BRANCH

Chief: James Wright

725-3616

803/725-3855

WASTE MANAGEMENT TECHNICAL SUPPORT BRANCH

Chief: Virgll Sauls

725-3616

$803 / 725-4505$

*DIAL AREA CODE

* RCRA/CERCLA/TSCA REGULATORY RESPONSE LEAD

SR - 2 
WESTINGHOUSE SAVANNAH RIVER COMPANY

SAVANNAH RIVER SITE

AIKEN, SOUTH CAROLINA 29808

P.O. BOX 616

AIKEN, SOUTH CAROLINA 29802

FAX*

725-4914

PRESIDENT: AMBROSE SCHWALLIE

\section{ENVIRONMENT}

ESH \& QA DIVISION

V.P. \& General Manager: Robert Campbell

ENVIRONMENTAL PROTECTION DEPARTMENT

Manager: Jack Roberts

\section{ENVIRONMENTAL RESTORATION}

ENVIRONMENTAL RESTORATION \& WASTE MANAGEMENT DIVISION

V.P. \& General Manager: Norman Boyter

ENVIRONMENTAL RESTORATION DEPARTMENT

Manager: Jim Pope

\section{WASTE MANAGEMENT}

ENVIRONMENTAL RESTORATION \& WASTE MANAGEMENT DIVISION

V.P. \& General Manager: Norman Boyter

WASTE MANAGEMENT DEPARTMENT

Manager: G. Todd Wright
$725-4943$

$803 / 725-4408$

725-4676

$803 / 725-1728$

$725-8310$

$803 / 725-2585$

644-6922

$803 / 644-6840$

$725-8310$

$803 / 725-2585$

577-0135

$803 / 557-0015$

*DIAL AREA CODE

**RCRA/CERCLA/TSCA REgULATORY RESPONSE LEAD

SR - 3 


\section{Schenectady Naval Reactors (SNR)}


SCHENECTADY NAVAL REACTORS OFFICE - DOE

KNOLLES ATOMIC POWER LABORATORY

RIVER ROAD

SCHENECTADY, NEW YOAK 12301

P.O. BOX 1069

SCHENECTADY, NEW YORK 12301

FAX*

$395-6390$

MANAGER: PHILIP SALM

ENVIRONMENT

RADIOLOGICAL ENVIRONMENTAL CONTROL \& SAFETY DIVISION Manager: Andrew Seepo ${ }^{\star \star}$

$395-6390$

$518 / 395-6366$

ENVIRONMENTAL RESTORATION

RADIOLOGICAL ENVIRONMENTAL CONTROL \& SAFETY DIVISION Manager: Andrew Seepo

$395-6390$

$518 / 395-6366$

\section{WASTE MANAGEMENT}

RADIOLOGICAL ENVIRONMENTAL CONTROL \& SAFETY DIVISION Manager: Andrew Seepo
$395-6390 \quad 518 / 395-6366$ 
STRATEGIC PETROLEUM RESERVE - DOE

PROJECT MANAGEMENT OFFICE

900 COMMERCE ROAD, EAST

NEW ORLEANS, LOUISIANA 70123

FAX* COMMERCIAL

PROJECT MANAGER: WILLIAM GIBSON

734-4427 504/734-4201

TECHNICAL ASSURANCE

ASSISTANT PROJECT MANAGER: CHUCK DOBSON

$734-4427 \quad 504 / 734-4274$

\section{ENVIRONMENT}

ENVIRONMENT, SAFETY \& HEALTH DIVISION

Director: Melissa Smith**

$734-4427$

$504 / 734-4387$

ENVIRONMENT

Manager: Sidney Evans

734-4427

$504 / 734-4353$

\section{ENVIRONMENTAL RESTORATION}

ENVIRONMENT, SAFETY \& HEALTH DIVISION

Director: Melissa Smith

734-4427

$504 / 734-4387$

ENVIRONMENT

Manager: Sidney Evans

$734-4427$

$504 / 734-4353$

\section{WASTE MANAGEMENT}

ENVIRONMENT, SAFETY \& HEALTH DIVISION

Director: Melissa Smith

$734-4427 \quad 504 / 734-4387$

ENVIRONMENT

Manager: David Brine

734-4427 504/734-4277

*DIAL AREA CODE

**RCRA/CERCLA/TSCA REGULATORY RESPONSE LEAD

$$
\text { SPR - } 1
$$


DYMMCDERMOTT PETROLEUM OPERATIONS (DPOC) 850 SOUTH CLEARVIEW PARKWAY

NEW ORLEANS, LOUISIANA 70123

FAX* COMMERCIAL

PROJECT MANAGER: CURTIS JOHNSON

734-4614 504/734-4917

ENVIRONMENT

ENVIRONMENTAL, SAFETY, AND HEALTH

Director: Charles Reith

734-4464

$504 / 734-4451$

ENVIRONMENTAL CONTROL

Manager: Vacant

$734-4464$

$504 / 734-4557$

ENVIRONMENTAL RESTORATION

ENVIRONMENTAL, SAFETY, AND HEALTH

Director: Charles Reith

$734-4464$

$504 / 734-4451$

ENVIRONMENTAL CONTROL

Manager: Vacant

734-4464

$504 / 734-4557$

\section{WASTE MANAGEMENT}

ENVIRONMENTAL, SAFETY, AND HEALTH

Director: Charles Reith

$734-4464 \quad 504 / 734-4451$

ENVIRONMENTAL CONTROL

Manager: Vacant

734-4464 504/734-4557

*DIAL AREA CODE

${ }^{*}$ RCRA/CERCLA/TSCA REGULATORY RESPONSE LEAD

SPR - 2 
Superconducting Super Collider (SSC) 
SUPERCONDUCTING SUPER COLLIDER PROJECT OFFICE (SSC) - DOE

OFFICE OF ENERGY RESEARCH

2550 BECKLEYMEADE AVE.

DALLAS, TEXAS 75237

FAX

708-2525

708-2504

708-2525

708-2504

ENVIRONMENTAL COMPLIANCE

Tony Robledo**

708-2525

708-2504

$708-2525$

708-2504

ENVIRONMENTAL COMPLIANCE

Joyce Stubblefield

ENVIRONMENTAL RESTORATION

COMPLIANCE DIVISION

Director: Nat Brown

708-2525

708-2504

\section{WASTE MANAGEMENT}

COMPLIANCE DIVISION

Director: Nat Brown

ENVIRONMENTAL COMPLIANCE

Tony Robledo
708-2525

708-2504

708-2525

708-2504
$214 / 708-2589$

$214 / 708-2513$ 214/708-2521

$214 / 708-2589$

$214 / 708-2552$

$214 / 708-2589$

$214 / 708-2513$ 
SUPERCONDUCTING SUPER COLLIDER LABORATORY (SSCL)

2550 BECKLEYMEADE AVENUE, SUITE 125

DALLAS, TEXAS 75237-3946

FAX*: COM 214/708-0000

FAX* COMMERCIAL

DIRECTOR: ROY SCHWITTERS

708-0000 214/708-1036

ENVIRONMENT

ENVIRONMENT, SAFETY \& HEALTH OVERSIGHT

Asst. Director: Larry Coulson

708-1524 214/708-1032

Assistant Head: Sam Baker

708-1524 214/708-1042

\section{ENVIRONMENTAL RESTORATION}

ENVIRONMENT, SAFETY \& HEALTH DEPARTMENT

Manager: Phil Shelley

708-1930 214/708-1057

Deputy Manager: Ben Reitze

708-1930 214/708-1922

\section{WASTE MANAGEMENT}

ENVIRONMENT, SAFETY \& HEALTH

Manager: Phil Shelley

708-1930

214/708-1057

Deputy Manager: Ben Rietze

708-1930 214/708-19222 

YUCCA MOUNTAN SITE CHARACTERIZATION PROJECT OFFICE (MMPO) - DOE

101 CONVENTION CENTER DRIVE

SUITE 200

LAS VECAS, NEVADA 89100

P.O. BOX 08009

LAS VECAS, NEVADA $80193-8008$

FAX COMMERCIAL

MANAGER: CARL GERTZ

794-7907

702/794-7920

794-7908

\section{ENMIRONMENT}

PROJECTS \& OPERATIONS CONTROL DIVISION

Director: Wendy Dixon*

794-7907

794-7908

702/794-7949

C.-ERATIONS CONTROL BPANCH

Chief: Vacant

794-7907

$794-7908$

\section{ENMRONMENTAL RESTORATION}

PROJECTS \& OPERATIONS CONTROL DIVISION

Director: Wendy Dixon

794-7907

794-7908

702/794-7949

OPERATIONS CONTROL BRANCH

Chiof: Vacant

794-7907

794-7908

\section{MASTE MANAGEMENT}

PROJECTS \& OPERATIONS CONTROL DIVISION

Director: Wendy Dbxon

794-7807

794-7908

702/794-7949

OPERATIONS CONTROL BRANCH

Chiff: Vacant

794-7907

794-7908

Physical Scientist: Vacant

794-750\%

794-7908

* DIAL AREa CODE

**RCRACERCLATSCA REGULATORY RESPONSE LEAD

$$
\text { YMPO - } 1
$$



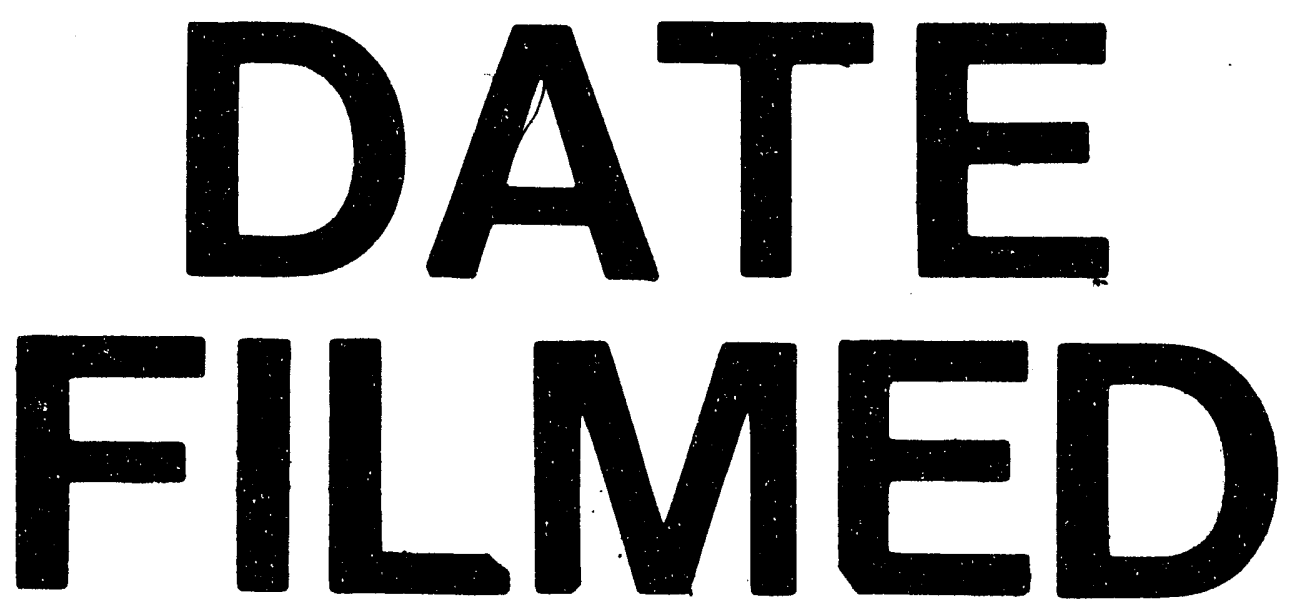

1

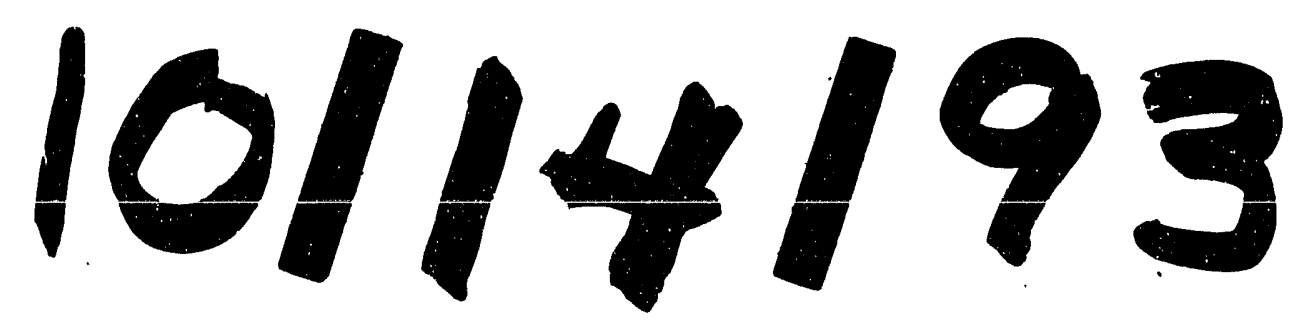


